\title{
LA-7384-MS
}

Informal Report

\section{A Catalog of Two-Dimensional Vortex Patterns}

莫

$\overleftarrow{0}$

$\geqslant$

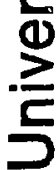

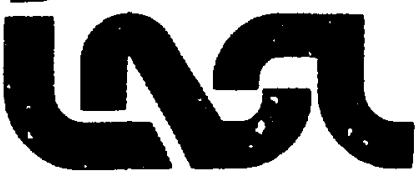


LA.7384-MS

Informal Report

Special Distribution

Issued: October 1978

\title{
A Catalog of Two-Dimensional Vortex Patterns
}

\author{
L. J. Campbell
}

Robert Ziff

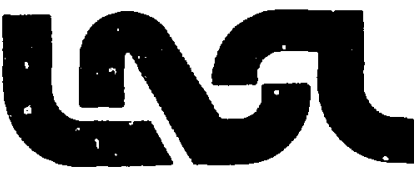


A CATALOG OF TWO-DIMENSIONAL VORTEX PATTERNS

by

L. J. Campbell and Robert Ziff

ABSTRACT

We list the two-dimensional patterns that correspond to minima in the free energy of identical parallel vortices inside a rotating cylinder of liquid such as superfluid ${ }^{4} \mathrm{He}$. All known stable patterns are listed for $\mathrm{N}=1,2, \ldots, 30,37,50$, where $N$ is the number of vortices. The two lowest energy patterns are shown for all "triangular" numbers, $N=1+6(1+2+3+.$.$) , through N=217$. For each value of $\mathrm{N}$ the different patterns are ordered according to their relative free energy.

\section{INTRODUCTION}

This report gives a more extensive and systematic list of two-dimensional stable vortex patterns than has been available heretofore. Although the results are directly applicable to studies of either the energy levels of rotating superfluid ${ }^{4} \mathrm{He}$ or dislocations in a single crystal under torsion, we use notation and terminology appropriate only to the former system.

Irreversible frictional effects (viscosity) drive normal fluids within a rotating container to a state of solid body rotation. However, the quantum nature of the superfluid component of liquid ${ }^{4} \mathrm{He}$ below $2.172 \mathrm{~K}$ absolutely precludes solid body rotation as a possible state of this component. Instead, superfluids react to rotating containers by generating a nearly uniform density of quantized rectilinear vortex lines, parallel to the axis of rotation, 
which simulate solid body rotation very closely on a distance scale large compared to the vortex spacing. However, for systems of only a few vortices, the discrete nature of the possible rotational states becomes manifest and permits an ordering of states on the basis of vortex number and pattern symmetry.

One hundred years ago interest in the vortex theory of atoms inspired Lord Kelvin to solve the case of three vortices equally spaced on a circle; five years later, J. J. Thomson proved that up to six vortices on a circle are stable. About fifty years later, T. H. Havelock ${ }^{1}$ proved that the presence of either an interior or exterior circular boundary caused absolute instability in a ring of seven or more vortices. More recently, Hess ${ }^{2}$ calculated the free energy of bounded rings consisting of up to seven vortices, both with and without an additional vortex at the center. For larger numbers of vortices the free energy formula becomes analyticaliy intractable and direct calculation is required. Stauffer and Fetter ${ }^{3}$, the first to report computer calculations of vortex patterns inside a circular boundary, published two patterns for 37 vortices together with their associated free energies for a particular angular velocity. We extend these results both in the number of patterns calculated and in the method of assigning a unique energy number to each pattern.

Section II contains the equations used to calculate the patterns and a description of the calculational procedure. A summary of the patterns is listed in Sec. III and figures of the patterns are shown in Sec. IV.

\section{EQUATIONS AND PROCEDURE}

In reduced units, the free energy per unit length of $N$ identical rectilinear vortices in a rotating cylinder is $\hat{z}$

$$
\begin{aligned}
f & =\ln \left[\frac{\prod_{i=1}^{N}\left(1-r_{i}^{2}\right) \prod_{i=1}^{N} \underset{j=1}{i-1}\left(1+r_{i}^{2} r_{j}^{2}-2 r_{i} r_{j} \cos \theta_{i j}\right)}{\prod_{i=1}^{N} \prod_{j=1}^{i-1}\left(r_{i}^{2}+r_{j}^{2}-2 r_{i} r_{j} \cos \theta_{i j}\right.}\right] \\
& -\omega \sum_{i=1}^{N}\left(1-r_{i}^{2}\right)+N \ln (R / a),
\end{aligned}
$$


where

$f=$ free energy in inits of $\rho k^{2} / 4 \pi$,

$r_{j}=$ radial distance of $j^{\text {th }}$ vortex from center of cyilinder (and axis of rotation) in units of $R$,

$\theta_{i j}=\theta_{i}-\theta_{j}$, the angle between $\underset{\sim}{r}$ and ${\underset{\sim}{r}}_{j}$,

$\omega=$ reduced angular velocity of cylinder, equal to $2 \pi R^{2} \Omega / k$ where $\Omega$ is the

physical angular velocity,

$\mathrm{a}=$ vortex core radius,

$p=$ density of liquid (for helium, $p$ is the superfluid density),

$k=$ strength of vortex (for helium, $k=h / m=0.990710^{-3} \mathrm{~cm}^{2} / \mathrm{s}$ ).

The term "free energy" is used because we are interested in the equilibrium states of the system for given angular velocity $\omega$ in analogy to the usual free energy $F$ which is appropriate to systems of given temperature, $F=$ $E$ - TS. That is, $f=e-w \ell$, where $e$ and $\ell$ are the dimensionless kinetic energy and the angular momentum of the system in the fixed, laboratory frame. Apart from a constant, $f$ is also the kinetic energy of the liquid in a reference frame that is rotating with angular velocity $w$. In these dimensionless units, solid-body rotation of the liquid has the free energy

$$
f_{s}=-\frac{1}{4} \omega^{2}
$$

Although the temperature is important in determining the time required for a helium vortex system to reach an equilibrium pattern, it does not affect the type of pattern nor the relative energy of different patterns and therefore can be ignored here.

A vortex pattern with coordinates $\left[r_{i}, \theta_{i}\right]$ is, respectively, stable or "nearly" stable if $f\left(r_{1}, \ldots, r_{N} ; \theta_{1}, \ldots, \theta_{N}\right)$ is a local minimum or a saddle point at these coordinates. In either case the pattern is stationary in the rotating frame. Beginning with an arbitrary configuration of vortices, an obvious way to find minima is to move the vortices successively toward lower energy configurations until a minimum is reached. The pattern may pass through one or more saddle points before it converges to a local minimum in the free energy. A simple algorithm to accomplish this consists of assigning to each vortex an effective velocity $u_{j}$ equal to the negative gradient of the free energy with respect to the coordinates of that vortex, $-\nabla_{j} f$. Allowing 
the vortices to move, in steps proportional to this effective velocicy, which changes after each step, results in a convergent pattern. This procedure works well except for angular velocities less than a critical value $\omega^{\star}(N)$ for which no free energy minima exist. For $N \leqslant 20$ we find $\omega^{\star}(N) \approx N+2$.

In deriving the velocity expression it is convenient to use complex numbers, $z=x+i y, \bar{z}=x-i y$, which allows the free energy to be written as

$$
\begin{aligned}
& f=\ln \left[\frac{\pi\left(1 \cdots\left|z_{j}\right|^{2}\right) \pi<j\left|1-z_{i} \bar{z}_{j}\right|^{2}}{\underset{i}{\pi<j}\left|z_{i}-z_{j}\right|^{2}}\right] \\
& -\omega\left\{\left(1-\left|z_{j}\right|^{2}\right)+N \text { ln }(R / a)\right. \text {. }
\end{aligned}
$$

The gradient transforms to complex notation as $\underline{\sim}_{j} f \rightarrow 2 \frac{\partial}{\partial z} f$ which gives the following expression for the pattern-converging velocity of the $j^{\text {th }}$ vortex,

$\frac{1}{2}\left(u_{j x}+i u_{j y}\right)=\sum_{\ell} \frac{1}{z_{j}-\bar{z}_{\ell}}-\sum_{\ell} \frac{1}{z_{j}-1 / z_{\ell}}-\omega z_{j}$,

where the prime on the first summation indicates that $\ell \neq j$. The first summation represents the influence of all the image vortices and the second summation gives the effect of all the other vortices on the $j^{\text {th }}$. This velocity field is exactly orthogonal to the velocity field $v$ in the rotating frame of a classical hydrodynamic vortex system which causes the vortices to move physically on an equipotential surface of $f\left(z_{j}\right): u \propto-i v$. To see this, recall that the velocity of a classical vortex is the stream velocity at its position. In dimensional units, $v$ is given by the derivative of the stream function

$$
\overline{v(z)}=-\frac{\partial}{\partial z} W(Z),
$$

which, for identical vortices inside a circular cylinder, is ${ }^{4}$

$W(Z)=i \frac{K}{2 \pi}\left[\sum_{\ell=1}^{N} \ln \left(z-z_{l}\right)-\sum_{\ell=1}^{N} \ln \left(R^{2}-z \bar{z}_{l}\right)\right]$.

To use a reference frame in which the boundary is at rest requires the addition to $W(Z)$ of the stream function for solid body rotation in the direction 
opposite to the laboratory rotation $-i \Omega|z|^{2}$, where $\Omega$ is the physical value of the angular velocity. The physical stream velocity at $z$ is, therefore,

$\overline{v(Z)}=-\frac{\partial}{\partial Z}\left[W(Z)-i \Omega|z|^{2}\right]$

which becomes, upon defining $z \equiv Z / R$ and $\omega \equiv z \pi R^{2} \Omega / k$,

$\frac{2 \pi R}{k} \quad v(\bar{z})=-i\left[\sum_{\ell} \frac{1}{z-z_{\ell}}-\sum_{\ell} \frac{1}{z-1 / \bar{z}_{\ell}}-\omega \bar{z}\right]$.

Taking the conjugate of the above equation and comparing with Eq. (3) confirms that $u(z) \propto-i v(z)$. (When $z=z_{j}$, the $j=\ell$ term must be omitted in the first sumation in Eq. (7) because a vortex does not contribute directly to its own velocity.) Any system of vortices whose motion follows only the classical velocity of Eq. (') can never converge to a stationary pattern in the rotating frame for the obvious reason that such motion is constrained to an equipotential surface of the free energy. However, the physical velocity $w$ of superfluid vortices has components parallel to both $v$ of Eq. (7) and $u$ of Eq. (3), the latter component arising from irreversib?e frictional dissipation that accompanies all relative motion between superfluid vortices and the normal component of superfluid helium. That is,

$$
w=\left(d_{1}-i d_{2}\right) v,
$$

where the relative magnitude of the positve real coefficients $d_{1}$ and $d_{2}$ depends on the temperature and the concentration of ${ }^{3} \mathrm{He}$ impurities. (Because of the quantum nature of the superfluid state, the integrity of the circulation of the vortices is unaffected by the above frictional effects.) Although there are no physical conditions for which $d_{1} / d_{2}=0$, we have no reason to expect that any stationary patterns calculated by an algorithm that assumes $d_{1} / d_{2}=0$ are inaccessible to the physical system for that reason.

We find that the $N$ and $w$ dependences of the free energy of stationary patterns as given by Eq. (1) or (2) can be expressed to good accuracy by a relatively simple analytic formula, namely, the free energy $f_{c}$ of a "continuum" consisting of vortices in a perfect triangular lattice (see Eq. (1) of Ref. 3), 
$f_{c}(N, \omega)=\frac{1}{2} N^{2}\left(\frac{3}{2}-\ln N\right)-\omega N+\frac{1}{2} N(N-1) \ln \omega$

$$
-N b+N \ln R / a \text {, }
$$

where $b=4.15041281 / \pi-\ln \sqrt{\pi}=0.748752485$. Using Eq. (8) we are able to express the free eriergies of patterns as a small difference $\Delta f(N, \omega) \equiv f-f_{c^{*}}$ Moreover, $\Delta f$ is an insensitive function of the angular velocity if the latter is not near the critical value $\omega^{\star}$; when $\omega$ increases, $\Delta f$ quickly reaches an asymptotic value characteristic of the given stationary pattern. Therefore, we may simply and uniquely specify a $\Delta f_{0}$ for each pattern by defining

$$
\Delta f_{0}(N) \equiv \lim _{\omega \rightarrow \infty} \Delta f(N, \omega)
$$

This asympcotic value for large $\omega$ is equivaient to ignoring the effects of images in the calculation of $f$ at any positive $\omega$. (For $\omega$ somewhat smaller than $\omega^{\star}(\mathrm{N})$ the radius of the vortex pattern will exceed the radius of the container, $r=1$; this is formally permissible in the absence of images.) A considerable saving of computation is thereby realized both in the calculation of the vortex velocities (the image sum in Eq. (3) can be omitted) and in the calculation of $\Delta f$. To derive the simplified form of $\Delta f$, substitute $r_{j}^{-} \sqrt{\omega / \omega}$ for $r_{j}$ in Eq. (1) (because the radii scale as $\sqrt{\omega} r_{j}=\sqrt{\omega} r_{j}^{-}$for moderately large $\omega$ ) and take the 1 imit as $\omega \rightarrow \infty$. This gives, after omitting the primes on $r_{j}$ for notational convenience,

$$
\begin{aligned}
\lim _{\omega \rightarrow \infty} f= & -\ln \frac{\pi}{j<i}\left(r_{i}^{2}+r_{j}^{2}-2 r_{j} r_{j} \cos \theta_{i j}\right) \\
& +\frac{1}{2} N(N-1) \ln \omega / \omega^{-}-N \omega+\omega^{-} \sum r_{j}^{2}+N \ln R / a .
\end{aligned}
$$


Therefore, using Eq. (8),

$$
\begin{aligned}
\Delta f_{0} \equiv & \lim _{\omega \rightarrow \infty} f-f_{c}=-\ln \underset{j<i}{\pi}\left(r_{i}^{2}+r_{i}^{2}-2 r_{i} r_{j} \cos \theta_{i j}\right)+\omega^{-} \Sigma r_{j}^{2} \\
& -\frac{1}{2} N^{2}\left(\frac{3}{2}-\ln N\right)-\frac{3}{2} N(N-1) \ln \omega^{-}+N b,
\end{aligned}
$$

where the positions ${\underset{\sim}{j}}_{j}$ are calculated for the positive, but otherwise arbitrary, angular velocity $\omega^{\prime}$.

The behavior of $\Delta f(N, \omega)$ and the scaling of $\sqrt{\omega} r$ as functions of $w$ are ilTustrated in Table I for $N=18$. Here $r_{18}\left(18_{1}\right)$ is the radius of the outermost vortex in the first (i.e., lowest energy) pattern of 18 vortices. The pattern 18 became unstable for $\omega \approx 19.9$, somewhat lower than for the pattern 187 . Already at $\omega=25, \Delta f$ is within $3 \%$ of its value at $\omega=\infty$. This relative insensitivity of $\Delta f$ on $w$ is emphasized in the fourth column. As might be expected, the free energy difference between patterns is even less sensitive to $w$; this is illustrated in the fifth column for patterns $18_{1}$ and $187^{\circ}$

The patterns were calculated by letting an initial vortex configuration develop in "time" steps $\Delta t$ according to $E q$. (3) until $\Delta f_{0}$ reached a constant value within $10^{-5}$. The positions of all vortices were changed simultaneously at each $\Delta t$ step where $\Delta t \approx 0.2 /(\omega-N)$ was used.

We began each pattern calculation with an initial configuration of rings of vortices, centered about the origin, at radii roughly consistent with the value of $w$ used. (We found that patterns of higher free eneryij were not produced efficiently when random arrays were used as the initial configuration.) The resulting convergent pattern was then compared with patterns previcusly obtained for the same $N$ and $\omega$ and, if different, was saved. 
TABLE I

VARIATION OF PATTERN FREE ENERGY AND SIZE

WITH ANGULAR VELOCITY

\begin{tabular}{|c|c|c|c|c|}
\hline$\underline{\omega}$ & $\Delta f\left(16_{1}\right)$ & $\sqrt{\omega} r_{18}(18)$ & $\Delta f_{0}-\Delta f(18)$ & $\Delta f\left(18_{7}\right)-\Delta f\left(18_{1}\right)$ \\
\hline$\infty$ & $0.25241^{a}$ & 3.515846 & 0 & 0.22487 \\
\hline 90 & 0.25241 & 3.515859 & -0.00000 & 0.22487 \\
\hline 80 & 0.25240 & 3.515868 & -0.00001 & 0.22486 \\
\hline 70 & 0.25240 & 3.515890 & -0.00001 & 0.22486 \\
\hline 60 & 0.25238 & 3.515943 & -0.00003 & 0.22485 \\
\hline 50 & 0.25232 & 3.516094 & -0.00009 & 0.22482 \\
\hline 40 & 0.25211 & 3.516637 & -0.00030 & 0.22474 \\
\hline 30 & 0.25068 & 3.519597 & -0.00173 & 0.22447 \\
\hline 25 & 0.24539 & 3.527177 & -0.05301 & 0.22463 \\
\hline $20^{c}$ & 0.18940 & 3.612616 & -0.06301 & $\ldots b^{b}$ \\
\hline
\end{tabular}

\section{PATTERN SUMMARY}

In Table II are listed the free energies $\Delta f_{j}$ and ring numbers of the patterns. The ring numbers are the number of vortices with radii that differ by less than 0.02 (except for $N>50$ where the criterion is 0.05 ). For example, 14 vortices form a pattern that is roughly (within 0.02 ) a ring of 4 inside a ring of 10 , making the ring number of this pattern $(4,10)$.

In principle, Table II can be used to "ind the net free energy of a specific pattern. Given the experimental conditions of cylinder radius $R$ and angular velocity $\Omega$, one merely adds $\Delta f_{o}$ of Table II to $f_{c}$ as calculated 
according to Eq. (8). (As shown in Table $I$, some accuracy will be lost if $w$ is near $\omega^{\star}$.) For example, if $R=0.5 \mathrm{~cm}$ and $\Omega=2 \pi / 60 \mathrm{~s}^{-1}$ (that is, $1 \mathrm{rpm}$ ) then $\omega=164.4934$ and, except for temperatures near the superfluid transition, $R / a=3.610^{7}$. From Eq. (8), a continuum pattern of 5 vortices would have (dimensionless) free energy $f_{c}=-689.5549$, so the net energies of the discrete patterns are $f\left(5_{1}\right) \approx f_{C}+\Delta f_{0}\left(5_{1}\right)=-689.4218$ and $f\left(5_{2}\right) \approx$ -689.1512. However, to calculate $f_{C}$ to an accuracy of only 0.1 , comparable to the larger differences between $\Delta f_{0}$ of the various patterns for a given $N$, one must know R/a and $\Omega$ to unrealistically high accuracy. In this example, the necessary accuracy is $0.02 \%$ for $R / a$ and $0.01 \%$ for $\Omega$. Consequertiy, the $\Delta f_{n}$ of Table II, like other fine structure energies, are more relevant to experiments of high relative precision than those of absolute accuracy.

If, for given experimental conditions, it is a question of which of all patterns has the lowest free energy, one must first find $N$ by minimizing $f_{C}(N, w)$ with respect to $N$. This results in a transcendental equation,

$$
0=N \ln N-N(1+\ln \omega)+\omega+1 / 2 \ln \omega+b-\ln R / a
$$

which can be formally solved for $N$ by iteration if a lies between the two 1 imits, $\omega_{1}<\omega<\omega_{2}$, where

$$
\omega_{1}+1 / 2 \ell n \omega_{1}=\ln R / a-b
$$

and

$$
\omega_{2}=\left(\frac{R}{a}\right)^{2} e^{-2 b} .
$$

The first limit requires a minimum angular velocity for any vorticity and the second limit prevents vortex densities so large that the vortex cores over1 ap. For the example used above, $\omega_{1}=15.3, w_{2}=2.8510^{14}$, and the minimum in $f_{c}$ occurs for $N=103$. The free energy $f_{c}$ for this optimum number of vortices differs appreciably from that $\left(f_{s}\right)$ for solid-body rotation, $\left(f_{s}-f_{c}\right) / f_{s}=0.25$, which shows that quantum effects are still important in patterns of 100 vortices. (For $\omega 10$ times 1 arger, the optimum vortex number is 16 times greater and the above ratio is reduced to 0.03.) 
TABLE II

FREE ENERGIES AND RING NUMBERS OF KNOWN

STABLE VORTEX PATTERNS

\begin{tabular}{|c|c|c|c|c|c|c|}
\hline$\underline{N}$ & Order & $\Delta f_{0}$ & & & \multicolumn{2}{|c|}{ Ring Numbers } \\
\hline 1 & 1 & -.00125 & 1 & & & \\
\hline 2 & 1 & .19065 & 2 & & & \\
\hline 3 & 1 & .14418 & 3 & & & \\
\hline 4 & 1 & .10740 & 4 & & & \\
\hline 5 & $\begin{array}{l}1 \\
2\end{array}$ & $\begin{array}{l}.13367 \\
.40365\end{array}$ & & 4 & & \\
\hline 6 & $\begin{array}{l}1 \\
2\end{array}$ & $\begin{array}{l}.21781 \\
.24927\end{array}$ & $\begin{array}{l}1 \\
6\end{array}$ & 5 & & \\
\hline 7 & 1 & .16749 & 1, & 6 & & \\
\hline 8 & 1 & .09454 & 1 & 7 & & \\
\hline 9 & $\begin{array}{l}1 \\
2 \\
3 x^{a} \\
4 x\end{array}$ & $\begin{array}{l}.19405 \\
.26694 \\
.26716 \\
.54366\end{array}$ & $\begin{array}{l}1, \\
2, \\
2, \\
3,\end{array}$ & $\begin{array}{l}8 \\
4, \\
3, \\
6,\end{array}$ & $\begin{array}{l}3 \\
4\end{array}$ & \\
\hline 10 & $\begin{array}{l}1 \\
2 \\
3\end{array}$ & $\begin{array}{l}.22433 \\
.225 \% 8 \\
.41705\end{array}$ & $\begin{array}{l}2, \\
2, \\
1\end{array}$ & $\begin{array}{l}4, \\
2, \\
9\end{array}$ & $\begin{array}{l}4 \\
4,\end{array}$ & 2 \\
\hline 11 & $\begin{array}{l}1 \\
2\end{array}$ & $\begin{array}{r}.24918 \\
.28859\end{array}$ & $\begin{array}{l}3, \\
2,\end{array}$ & $\begin{array}{l}8 \\
9\end{array}$ & & \\
\hline 12 & $\begin{array}{l}1 \\
2 x \\
3\end{array}$ & $\begin{array}{l}.19344 \\
.19837 \\
.35022\end{array}$ & $\begin{array}{l}3, \\
3, \\
4,\end{array}$ & $\begin{array}{l}3, \\
6, \\
8\end{array}$ & $\begin{array}{l}6 \\
3\end{array}$ & \\
\hline 13 & $\begin{array}{l}1 \\
2\end{array}$ & $\begin{array}{r}.22432 \\
.24636\end{array}$ & $\begin{array}{l}4, \\
3,1\end{array}$ & $\begin{array}{r}9 \\
10\end{array}$ & & \\
\hline 14 & $\begin{array}{l}1 \\
2\end{array}$ & $\begin{array}{r}.17790 \\
.36713\end{array}$ & $\begin{array}{l}4, i \\
5,\end{array}$ & $\begin{array}{r}10 \\
9\end{array}$ & & \\
\hline 15 & $\begin{array}{l}1 \\
2 \\
3\end{array}$ & $\begin{array}{l}.23413 \\
.24711 \\
.24773\end{array}$ & $\begin{array}{ll}4, & 1 \\
5, & 1 \\
5, & 1\end{array}$ & $\begin{array}{l}11 \\
10 \\
10\end{array}$ & & \\
\hline
\end{tabular}




$\begin{array}{lllllll}16 & 1 & .21959 & 5, & 11 & & \\ & 2 & .36491 & 1, & 5, & 10 & \\ & 3 \times & .38647 & 1, & 5, & 5, & 5 \\ & 4 & .39842 & 4, & 4, & 8 & \\ & 5 & .40541 & 4, & 8, & 4 \\ 17 & 1 & .28394 & 1, & 5, & 11 \\ & 1 & .29412 & 5, & 12, & \\ & 3 & .34894 & 3, & 3, & 11 & \\ & 4 & .39682 & 1, & 6, & 10 & \\ & 5 & .47407 & 1, & 4, & 4, & 8\end{array}$

$\begin{array}{lllll}18 & 1 & .25241 & 1, & 6,11 \\ & 2 & .28316 & 1, & 5,12 \\ 3 & .35110 & 3, & 3,12 \\ 4 & .35215 & 6,12,12 \\ 5 & .35241 & 3, & 3,12 \\ 6 & .35626 & 6,12, & \\ 7 & .47728 & 5,13 & \end{array}$

$\begin{array}{llllll}19 & 1 & .18626 & 1,6,6,6 \\ 2 & .19317 & 1,6,12 \\ 3 & .33323 & 1, & 7,11 \\ 4 & .38632 & 1, & 5,13 \\ 5 & .45481 & 3, & 3,13\end{array}$

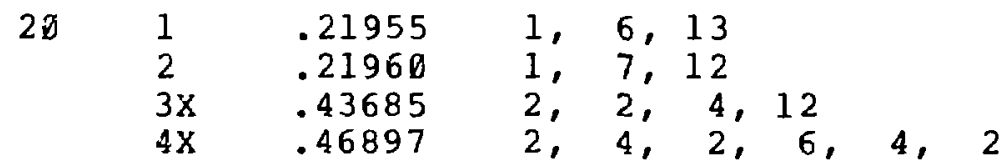

$\begin{array}{llllll}21 & 1 & .19096 & 1, & 7,13 \\ & 2 & .34793 & 1, & 6, & 6, \\ & .37628 & 1, & 4, & 4, & \\ & 3 & .392 & \\ 4 & .3950 & 2, & 2, & 2, & 3,12\end{array}$

$\begin{array}{lllll}1 & .25122 & 1,7,7, & 1 \\ 2 \times & .26311 & 1,7,14 \\ 3 & .36426 & 1,7,13 \\ 4 & .33339 & 2,7,13\end{array}$

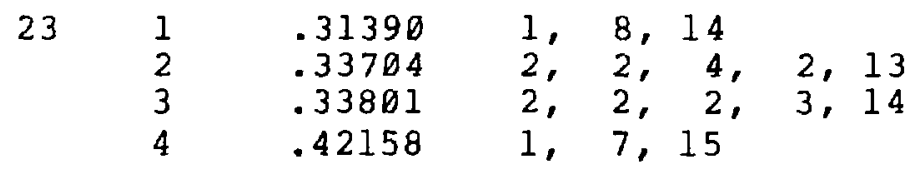

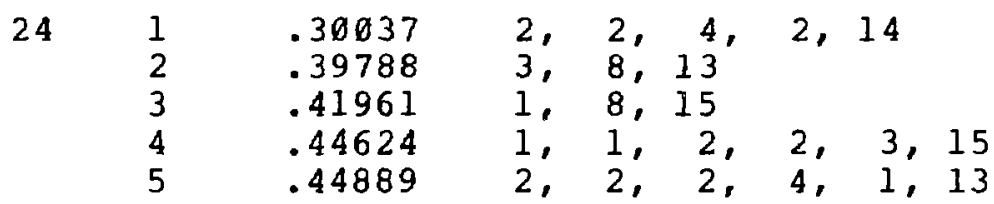




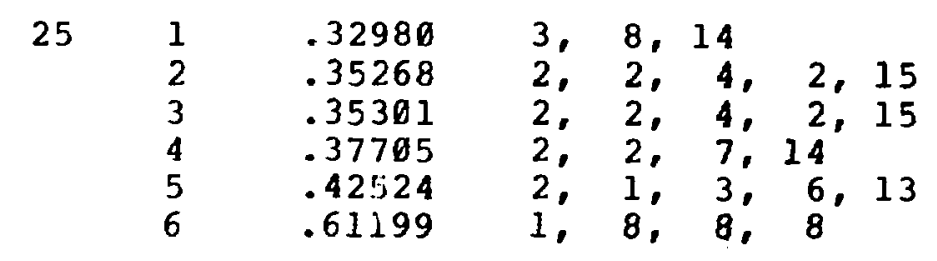

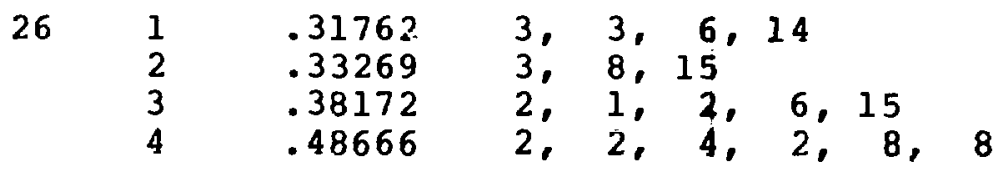

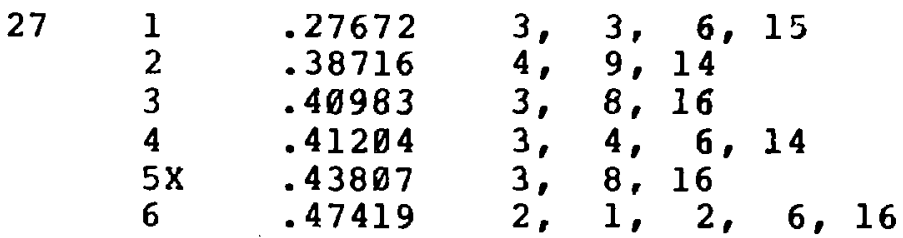

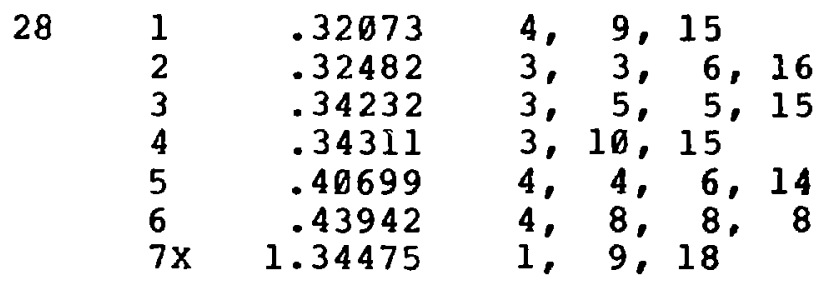

$\begin{array}{lllll}29 & 1 & .31163 & 4,10,15 \\ & 2 & .32340 & 4, & 9,16 \\ 3 & .34598 & 3, & 3, & 7,16 \\ & 4 & .45988 & 3, & 3,\end{array}$

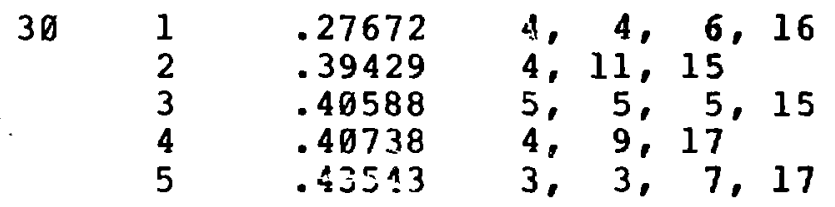

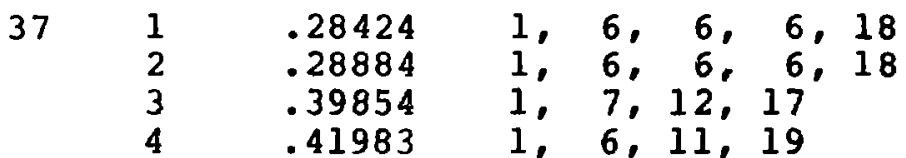

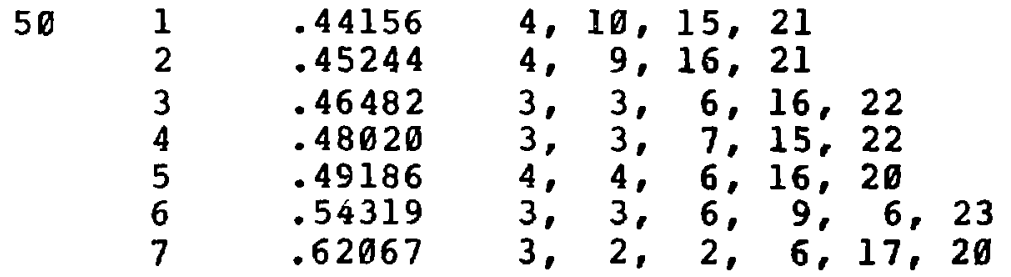




\begin{tabular}{|c|c|c|c|c|c|c|c|c|c|}
\hline 61 & $\frac{1}{2}$ & $\begin{array}{r}.39758 \\
.42173\end{array}$ & $\begin{array}{l}1, \\
1,\end{array}$ & $\begin{array}{l}6,12, \\
6,12,\end{array}$ & $\begin{array}{l}18, \\
18,\end{array}$ & $\begin{array}{l}24 \\
24\end{array}$ & & & \\
\hline 91 & $\begin{array}{l}1 \\
2\end{array}$ & $\begin{array}{l}.53680 \\
.58631\end{array}$ & $\begin{array}{l}1, \\
1,\end{array}$ & $\begin{array}{l}6,12, \\
6,12,\end{array}$ & $\begin{array}{l}18 \\
18\end{array}$ & $\begin{array}{l}24 \\
24\end{array}$ & $\begin{array}{l}30 \\
30\end{array}$ & & \\
\hline 127 & $\begin{array}{l}1 \\
2\end{array}$ & $\begin{array}{r}.70345 \\
.78294\end{array}$ & $\begin{array}{l}1 \\
1\end{array}$ & $\begin{array}{l}6,12, \\
6,12,\end{array}$ & $\begin{array}{l}18, \\
18,\end{array}$ & $\begin{array}{l}24, \\
24,\end{array}$ & $\begin{array}{l}30, \\
30,\end{array}$ & $\begin{array}{l}36 \\
36\end{array}$ & \\
\hline 169 & $\begin{array}{l}1 \\
2\end{array}$ & $\begin{array}{r}.89830 \\
1.01167\end{array}$ & $\begin{array}{l}1, \\
1,\end{array}$ & $\begin{array}{l}6,12, \\
6,12,\end{array}$ & $\begin{array}{l}18, \\
18\end{array}$ & $\begin{array}{l}24, \\
24,\end{array}$ & $\begin{array}{l}30, \\
30,\end{array}$ & $\begin{array}{l}36, \\
36,\end{array}$ & $\begin{array}{l}42 \\
42\end{array}$ \\
\hline 217 & $\begin{array}{l}1 \\
2\end{array}$ & $\begin{array}{l}1.12214 \\
1.27248\end{array}$ & $\begin{array}{l}1, \\
1,\end{array}$ & $\begin{array}{l}6,12, \\
6,12,\end{array}$ & $\begin{array}{l}18, \\
18,\end{array}$ & $\begin{array}{l}24, \\
24,\end{array}$ & 30, & $\begin{array}{l}36, \\
36\end{array}$ & $\begin{array}{ll}42, & 48 \\
42, & 48\end{array}$ \\
\hline
\end{tabular}

${ }^{a} x$ denotes patterns that are nearly stable.

As $N$ increases, the pattern with lowest energy changes from 1 vortex in the center to $2,3,4,5$, and then back to $1(N=6), 2(N=10), 3,4,5$, and again, $1(N=17)$. Having 1 vortex in the center of the lowest energy pattern is a property of vortex numbers close to "triangular" ring numbers $N=1+6$ $(1+2+3+\ldots)$, that is, $N=1,7,19,37,61,91,127,169,217$, etc.

\section{PATTERN FIGURES}

The patterns are illustrated in the Catalog below in order of increasing vortex number $N$ and, for each $N$, in order of increasing $\Delta f_{0}$, which is the asymptotic free energy difference with respect to the continuum model as wro . We show all the stable patterns we have found for $N: \leqslant 30$ and $N=37,50$. (Patterns $37_{2}$ and $37_{3}$ were shown by Stauffer and Fetter. ${ }^{3}$ ) In addition we include a few of the many nearly stable patterns, which are identified by an " $X$ " in the upper right-hand coiner. For $N=61,91,127,169,217$ only the two lowest energy patterns are shown. 
In addition to $\Delta f_{0}$, the ring numbers (defined in Sec. III) are also listed below each pattern. A result of the fine discrimination applied to patterns with $N \leqslant 50$ is ring numbers that are, in several cases, expanded beyond what one sees iy cursory inspection. For instance, pattern $9_{2}$ has ring numbers $(2,4,3)$ although on a coarser scale they would obviously be $(2,7)$. The $2 \%$ criterion permits urique ring numbers to most patterns that are similar, such as $9_{2}$ and $9_{3}$, but still not all, such as $15_{2}$ and $15_{3}$.

As explained in Sec. II, the absence of image effects makes $\Delta f_{0}$ independent of the value of angular velocity w used to calculate the pattern. The pattern size, however, does depend on $\omega$ and an accurate estimate of the radi $i$ of the various rings constituting a pattern can be derived from the exact second-moment relation that every stationary (stable or nearly stable) pattern in an unbounded fluid must satisfy $y^{5}$ :

$\omega \sum_{j=1}^{N} r_{j}^{2}=\frac{3}{2} N(N-1)$.

Assuming the pattern consists of $M$ rings of radii $r_{1}, \ldots, r_{M}$, each containing $N_{1}, \ldots, N_{M}$ vortices, respectively, the ring radii satisfy

$\omega r_{j}^{2}=\frac{2}{2}\left(N_{j}-1\right)+N_{1}+N_{2}+\ldots+N_{j-1}, \quad 1 \leqslant j \leqslant N$.

This formula is most useful for rings that do not deviate appreciably from circles. In Table III are listed the values of w used here.

TABLE III

ANGULAR VELOCITY USEO IN PATTERN CALCULATIONS

N

1 through 15

16 through 30

37

50

61

91

127

169

217

$$
\begin{aligned}
& \stackrel{\underline{\omega}}{N+10} \\
& N+8
\end{aligned}
$$

50

65

80

125

185

250

330 
The patterns for $N=61,91,127,169,217$ are included to illustrate the iendencies of those patterns which most closely approximate triangular syminetry which is known to be the preferred pattern for an infinite number of vortices. However, fur each of these $N$, the pattern $N_{1}$ of lowest free energy has less triangular symmetry than $\mathrm{N}_{2}$. In addition, the triangular lattices of both $\mathrm{N}_{1}$ and $\mathrm{N}_{2}$ suffer circular distortion that penetrates deeply into the patterns. (This is most easily seen by looking obliquely at the figures.) Because these pattorns are calculated in the absence of images they show that a finite pattern of vortices, even in an infinite container, displays triangular symmetry in less than half of its total area. This circular distortion results in a free energy that is greater than the (continuum model) value for an equal number of vrrtices in an infinite and perfect triangular lattice. This additional energy per vortex a ppears to be approaching a limiting value as $N$ increases: $\Delta f_{0}(N) / N=0.00590,0.00554,0.00532$, 0.00517 for $N=91,127,169$, and 217 , respectively. For comparison, the extra energy per vortex in a perfect square lattice is 0.01058 . Although the deviation $\Delta f_{0}$ of the free energy with respect to the continuum model increases with larger $N$, the ratio of this deviation to the total free energy decreases rapidly.

The nearly stable patterns are shown here to serve as a cautionary sample of patterns that may initially show excellent convergence, both spatially and in the ir second moment, Eq. (13), but are not subsequently stable. This is especially true of many patterns derived from initial vortex configurations of high symmetry such as those having ring numbers $(m, 2 m)$ or $(m, 3 m)$ or $(1, m, 2 m)$, etc. The free energy of these patterns is at a saddle-point and continued numerical iteration of the pattern around the apparent stable configuration will lead to the injection, through computer round-off error, of finite amplitude into the unstable modes of the pattern which then grow, leading to a new stable (or perhaps nearly stable) pattern. The acquisition of nearly stable patterns can be largely avoided by sightly randomizing the initial configuration. 


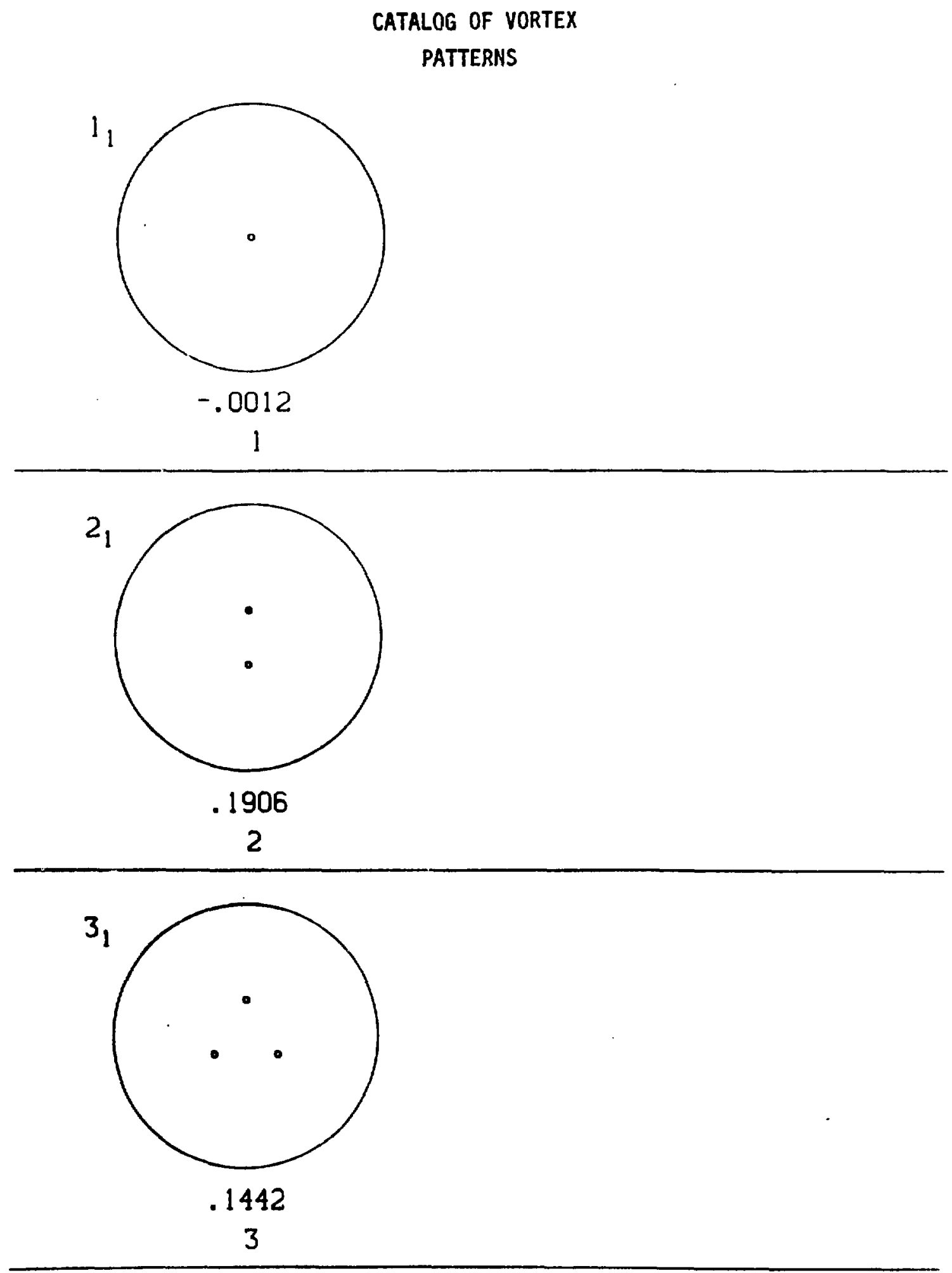



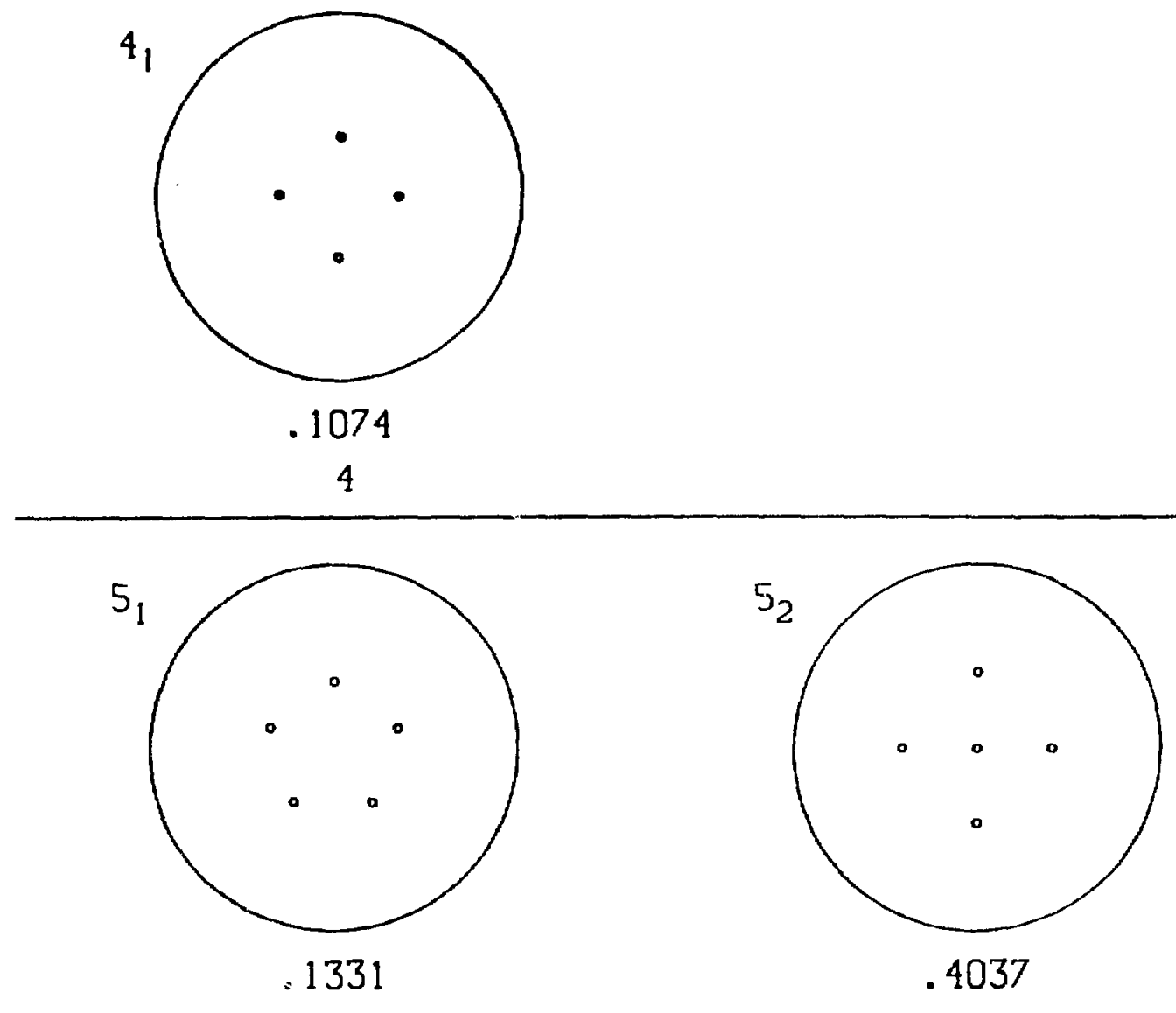

5

14
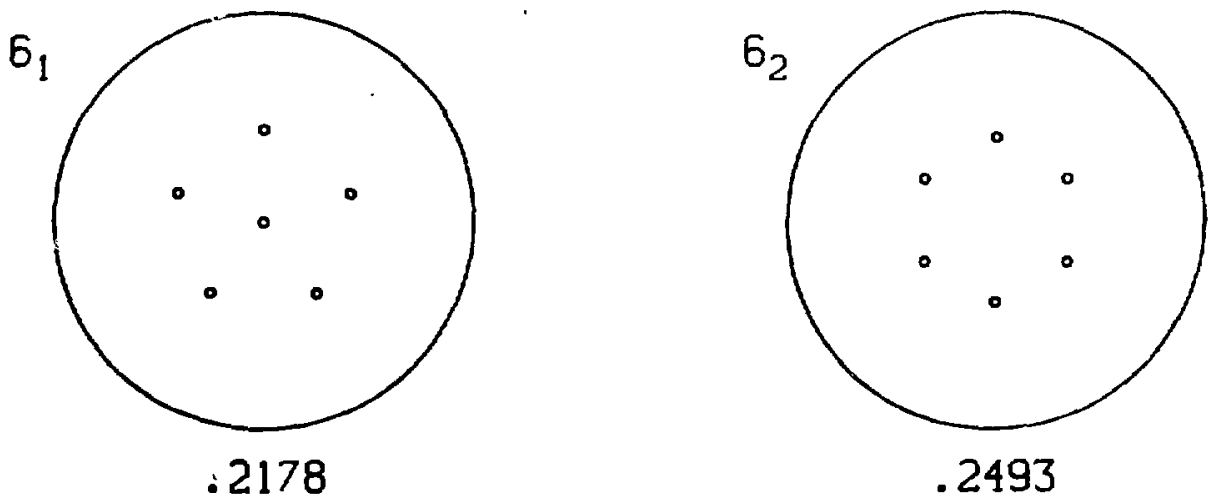

15

6 

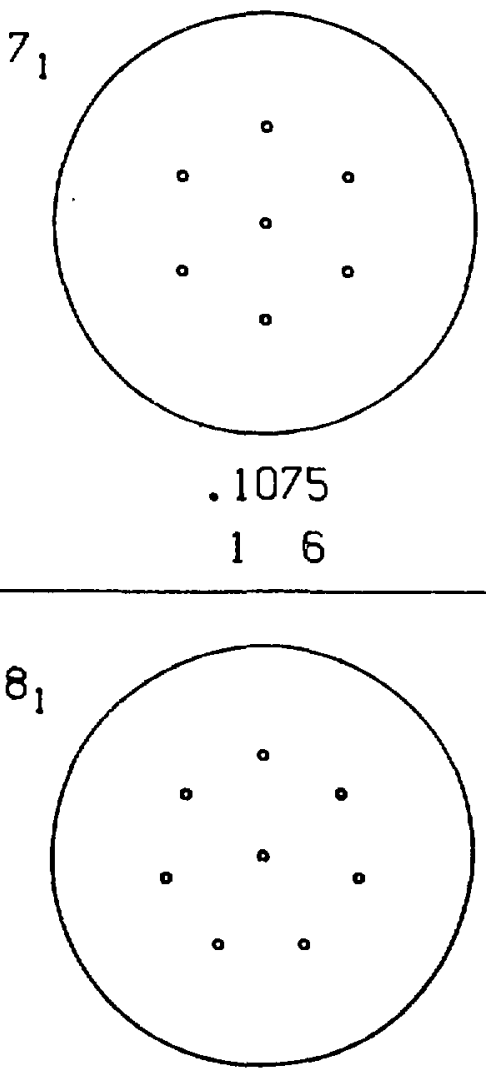

.0945

$9_{1}$

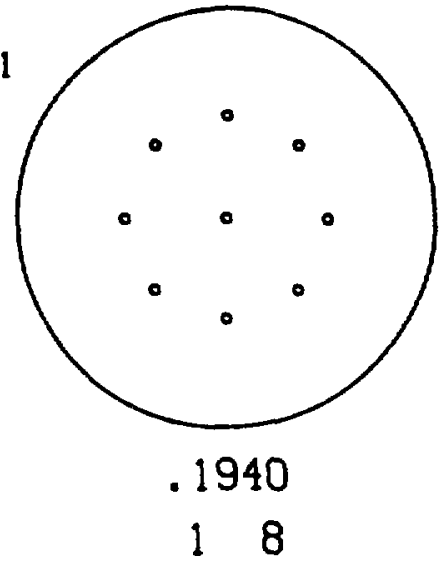

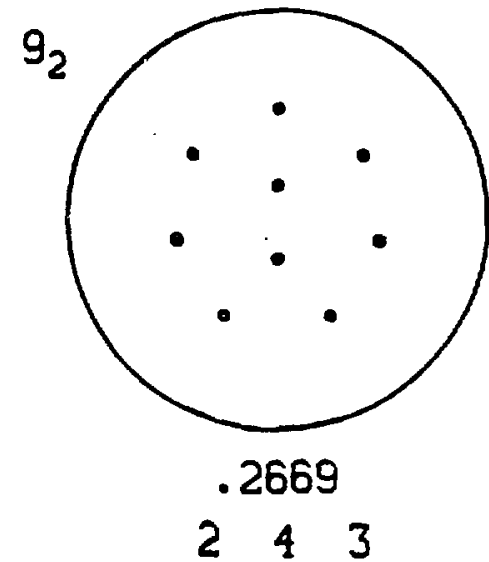



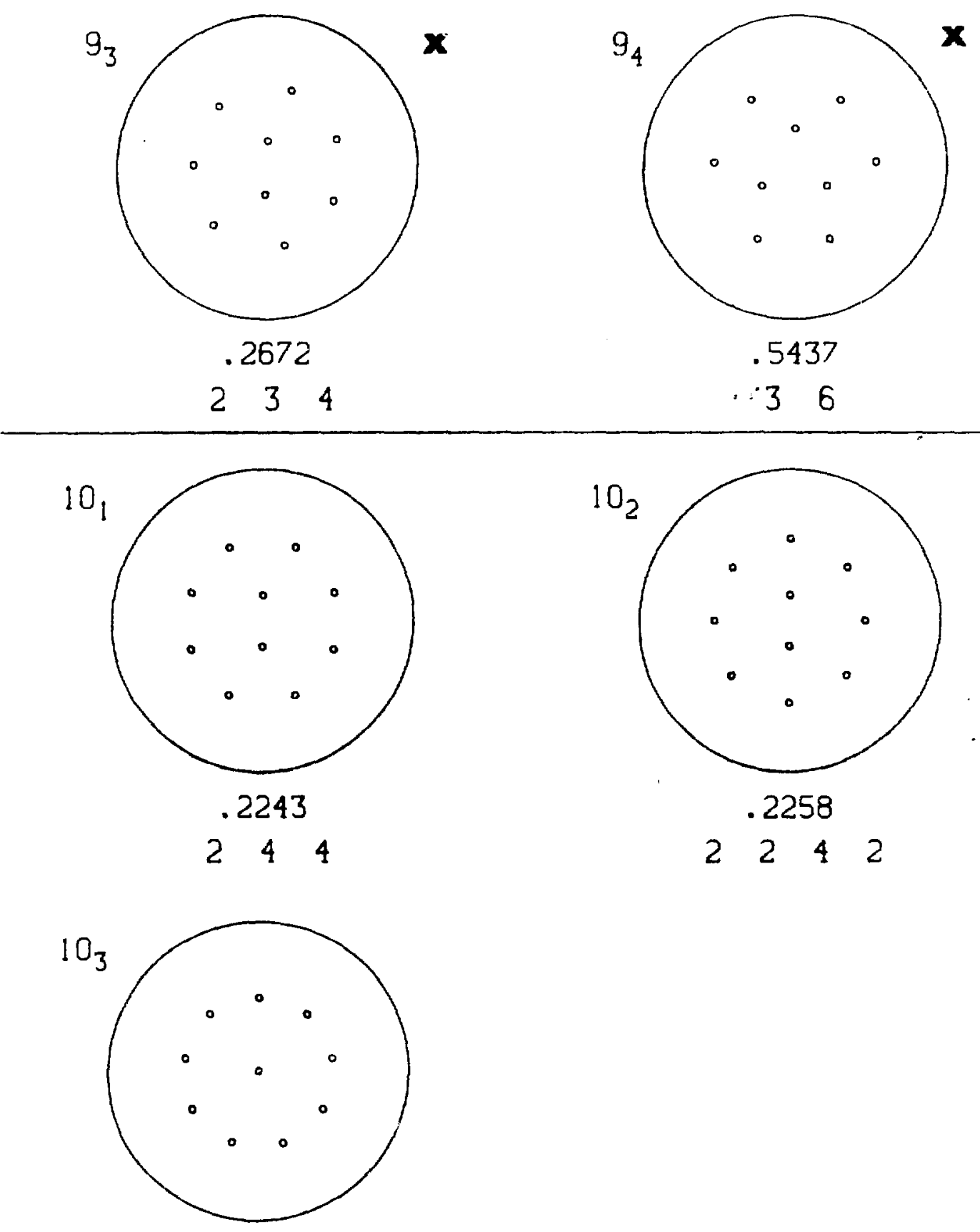

.4170

19 

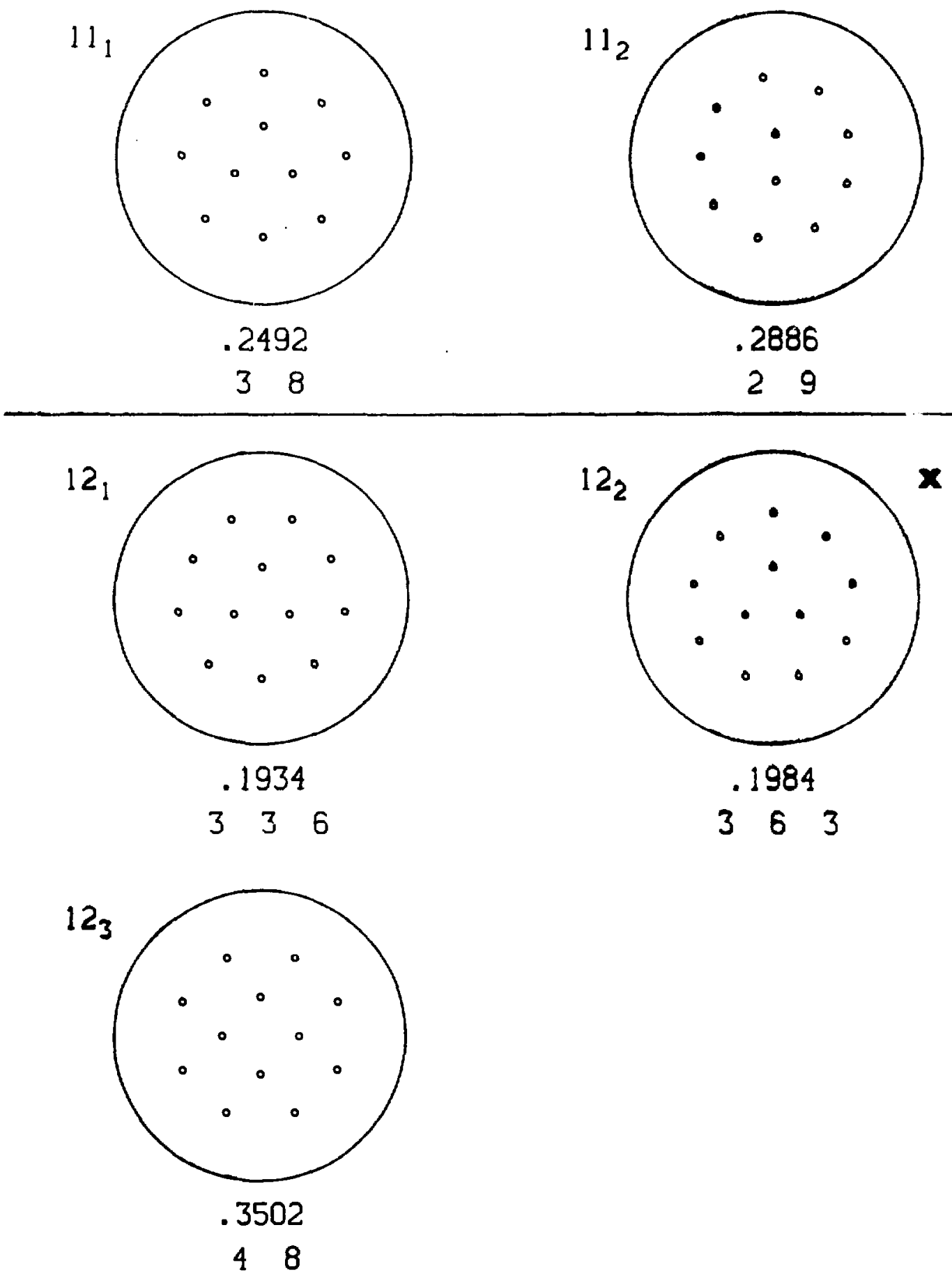

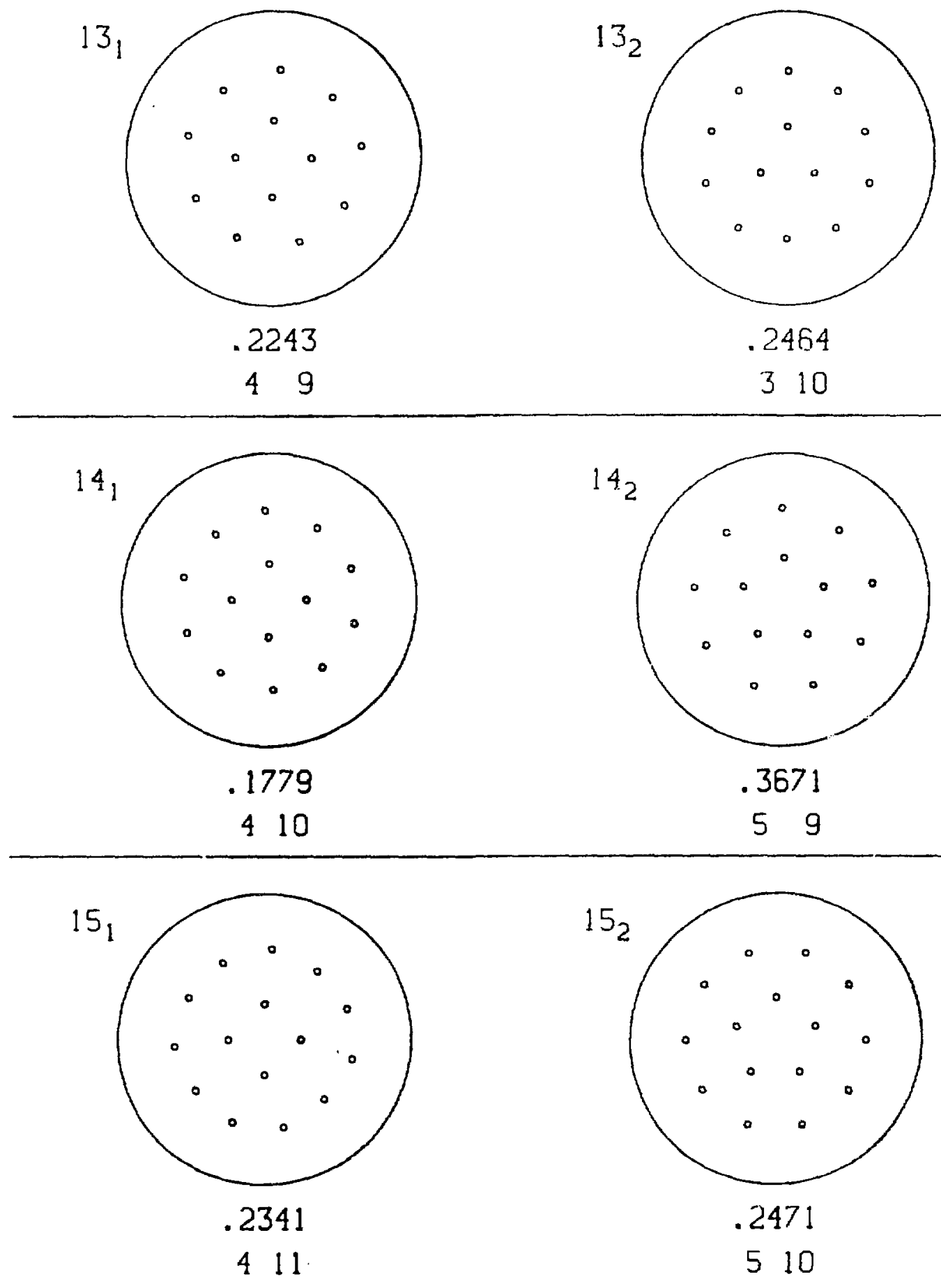

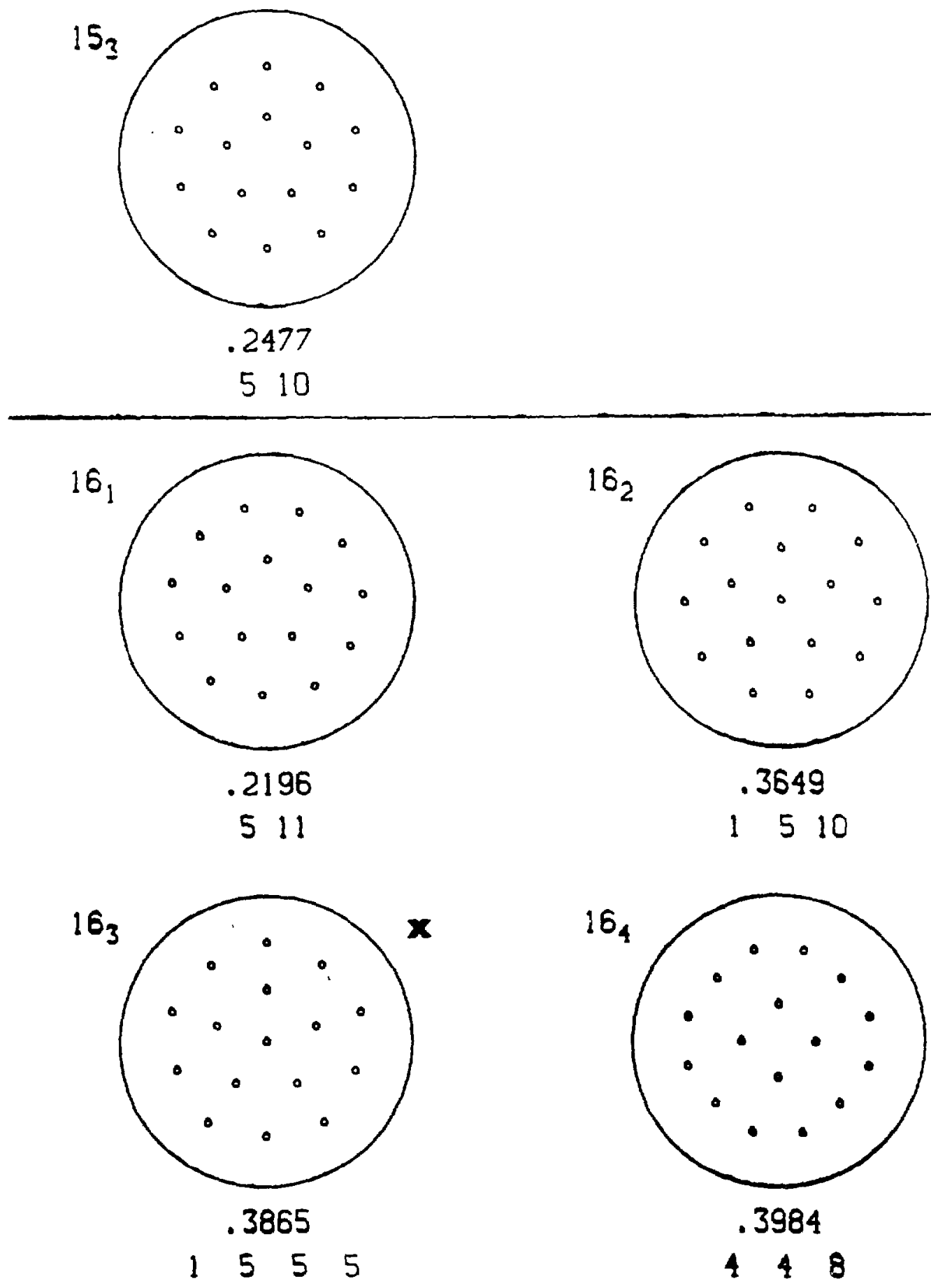

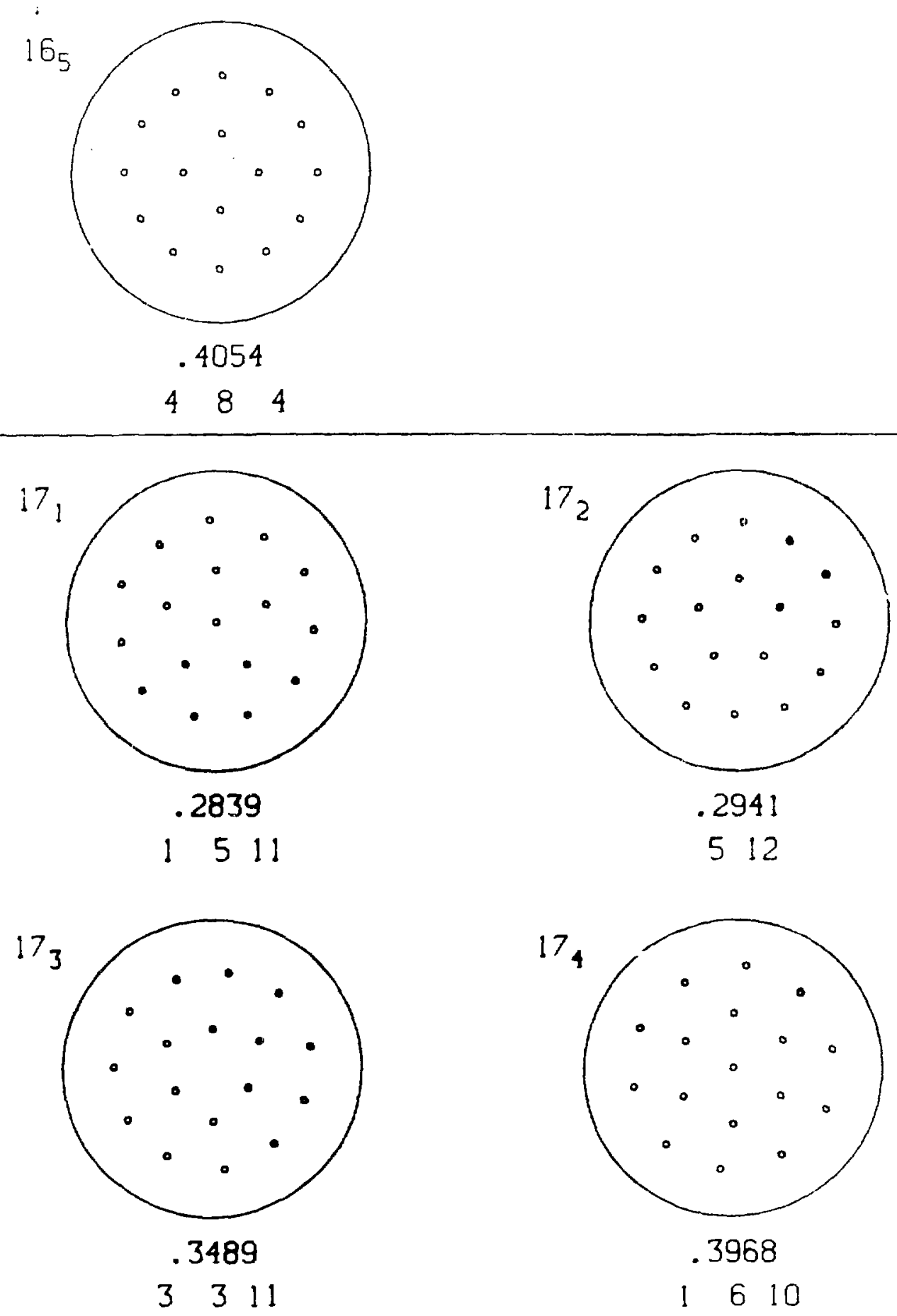



$18_{2}$
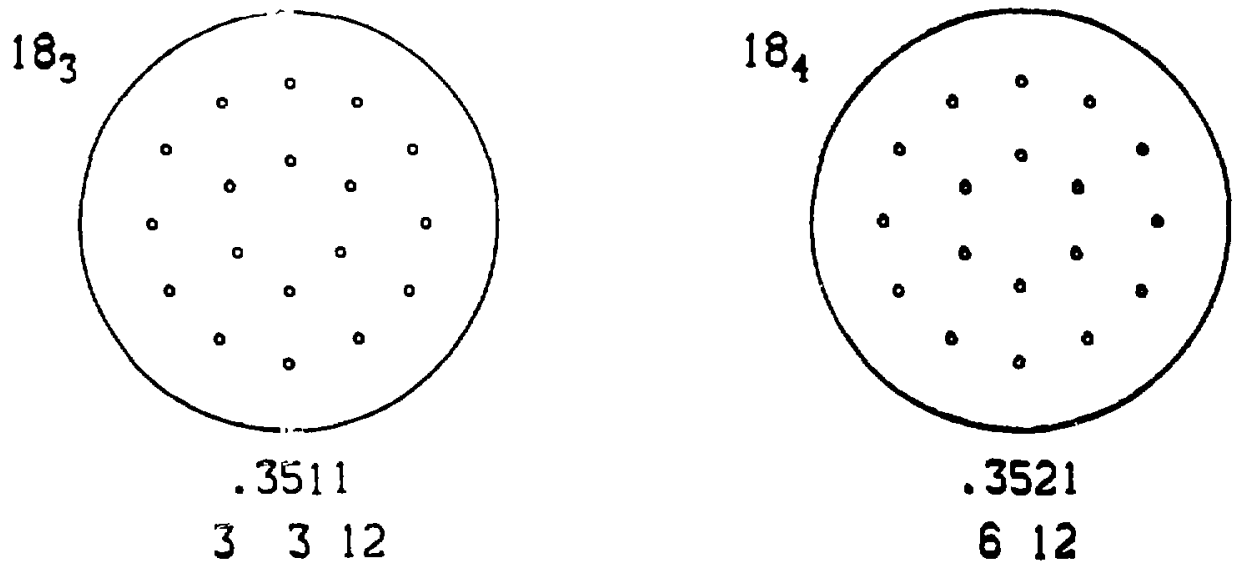

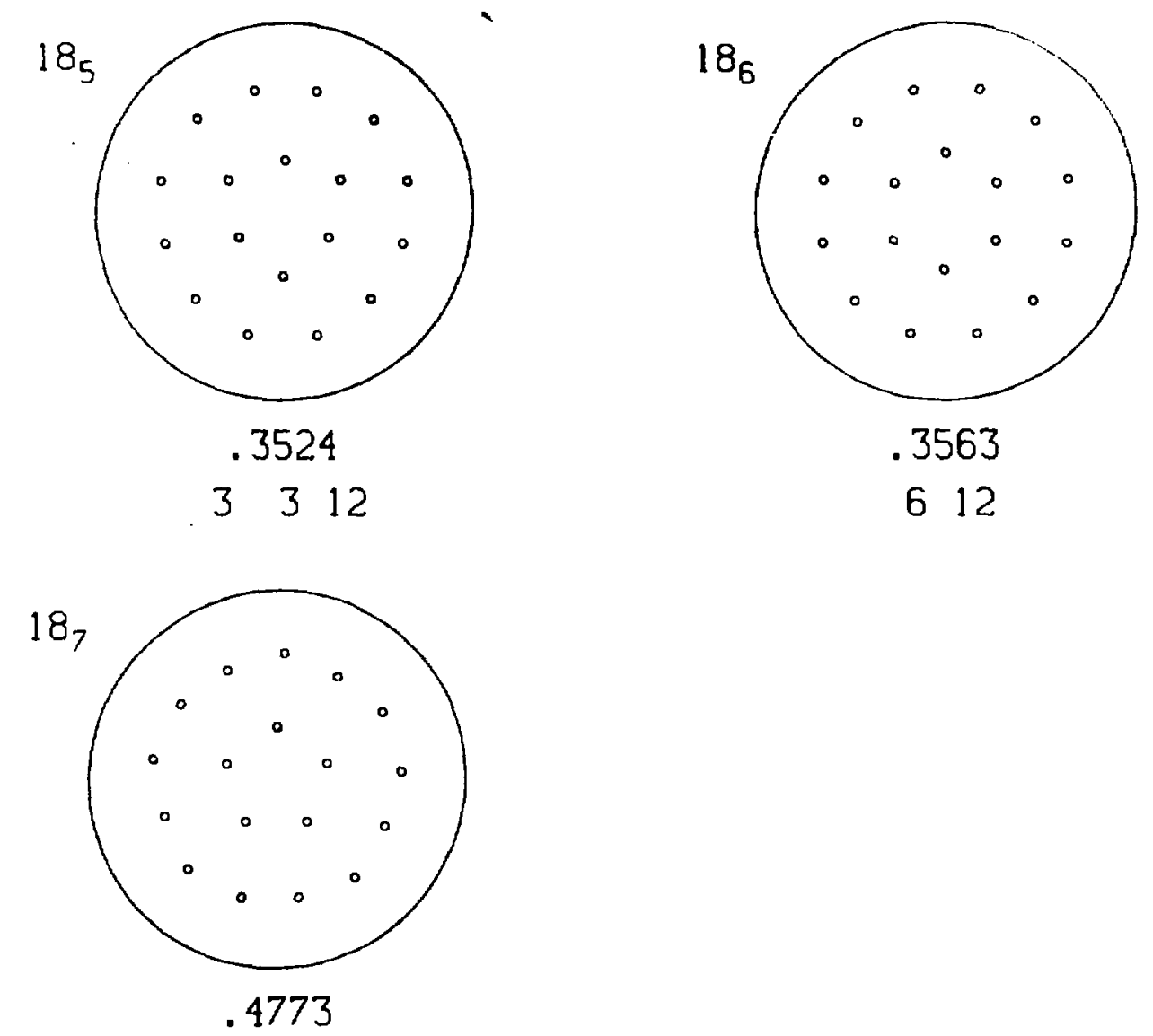

513
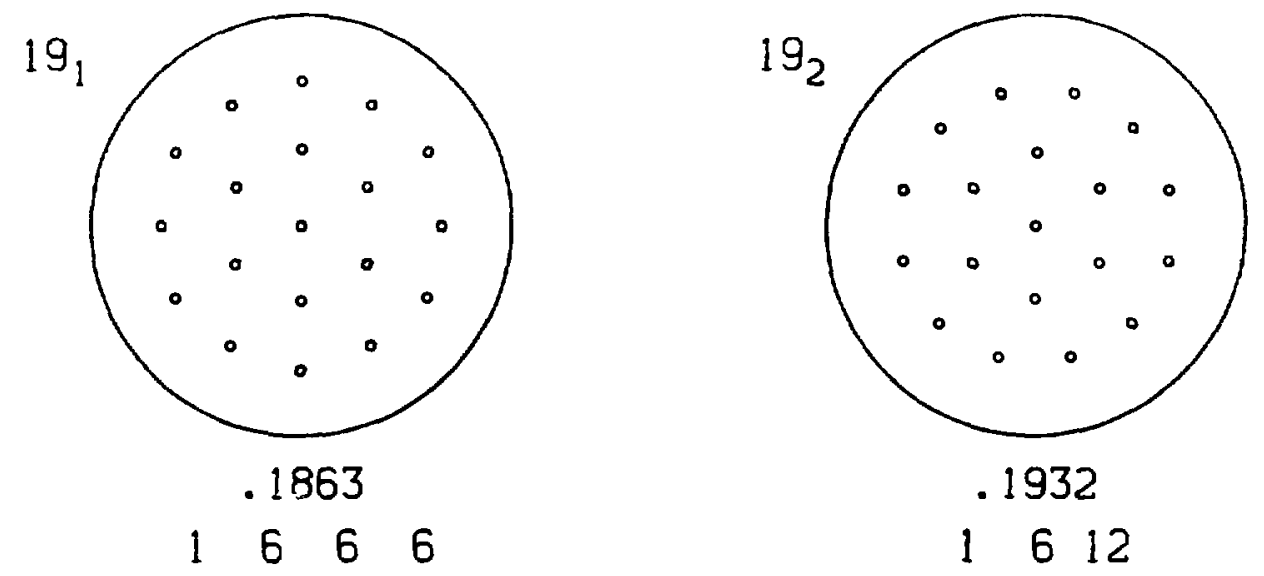
$19_{3}$

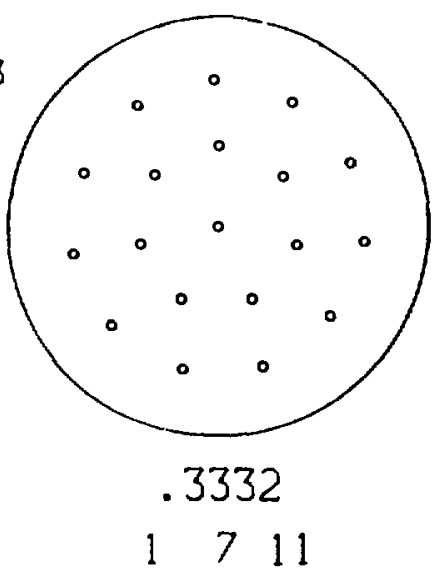

$19_{5}$

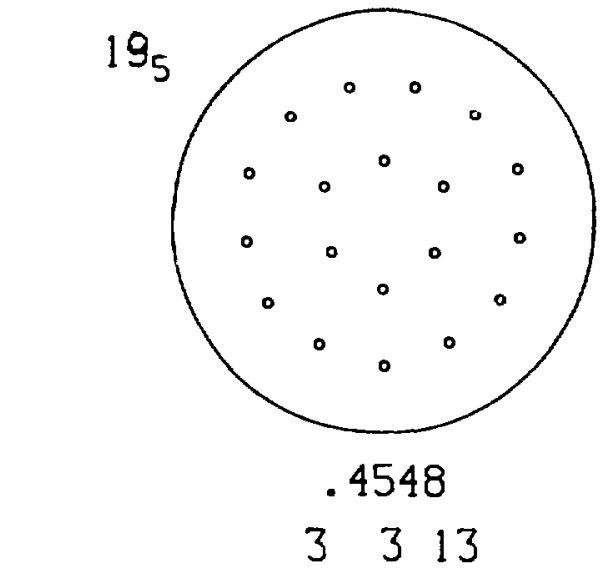

19

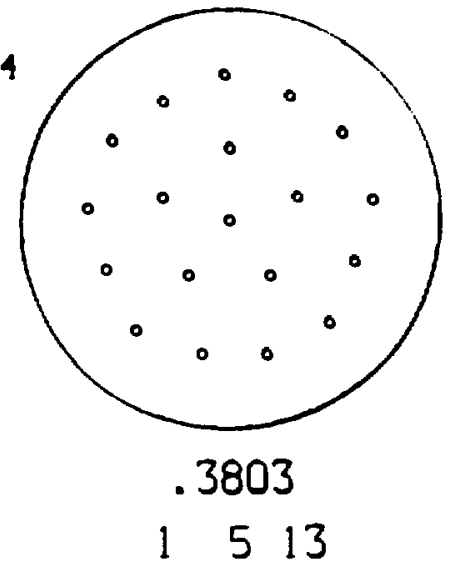

$2 \mathrm{O}_{1}$

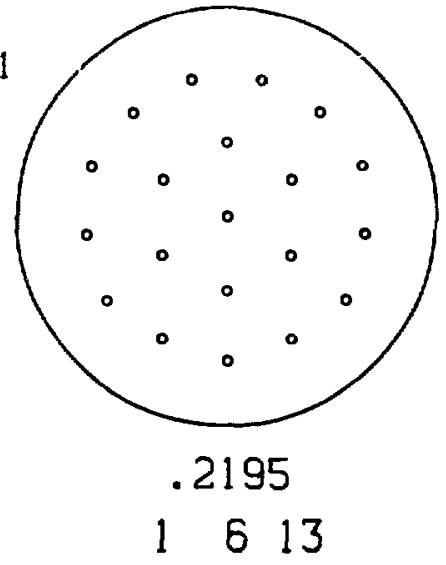

$2 \mathrm{O}_{2}$

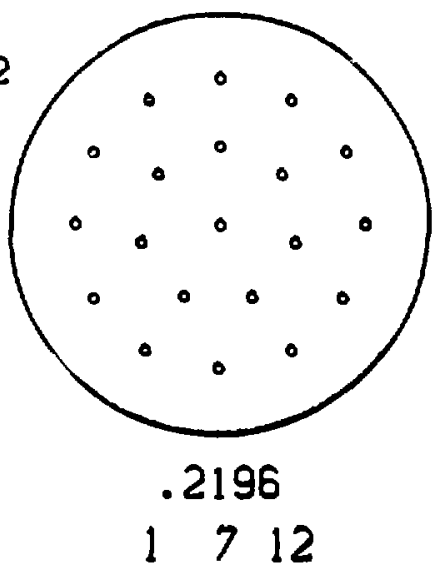


$20_{3}$

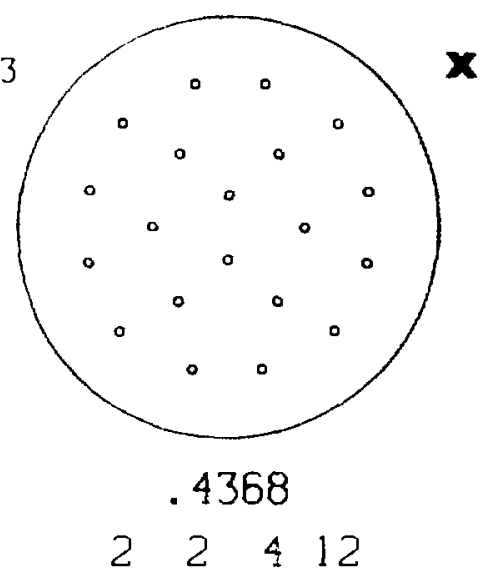

21

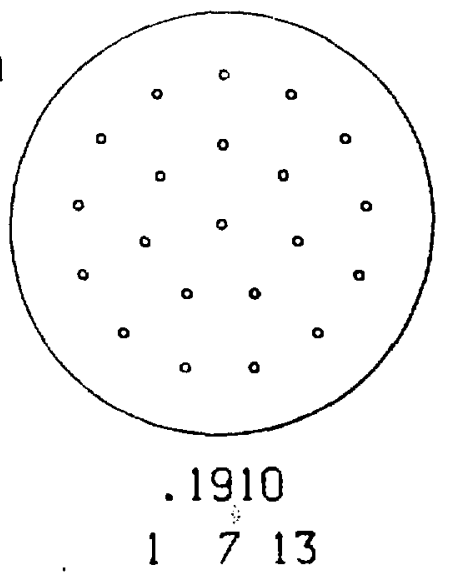

$21_{3}$

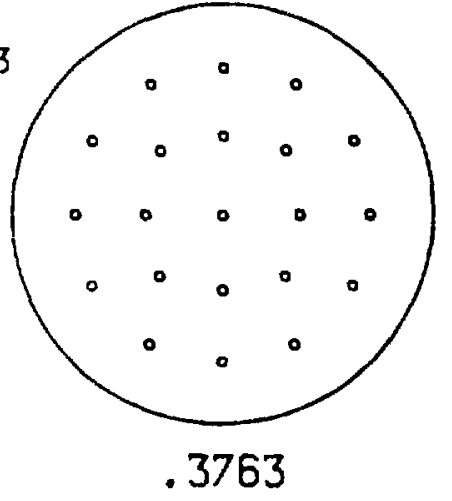

$\begin{array}{llll}1 & 4 & 4 & 12\end{array}$

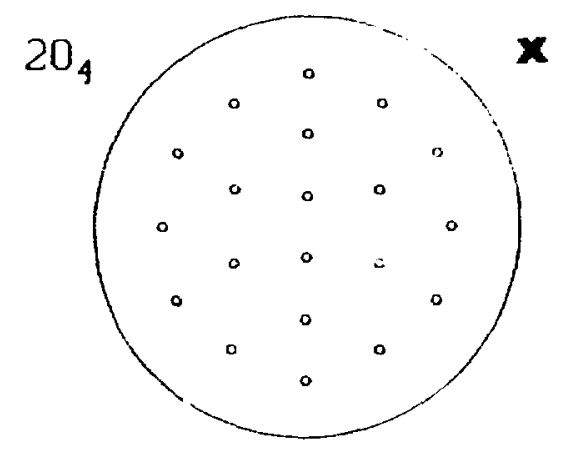

.4690

\section{$\begin{array}{llllll}2 & 4 & 2 & 6 & 4 & 2\end{array}$}
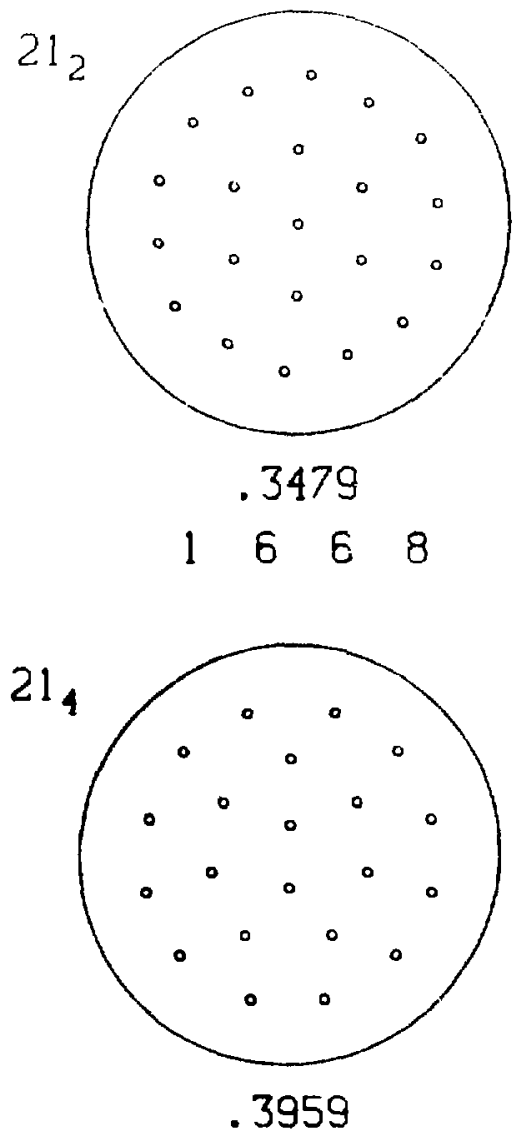

$\begin{array}{lllll}2 & 2 & 2 & 3 & 12\end{array}$ 

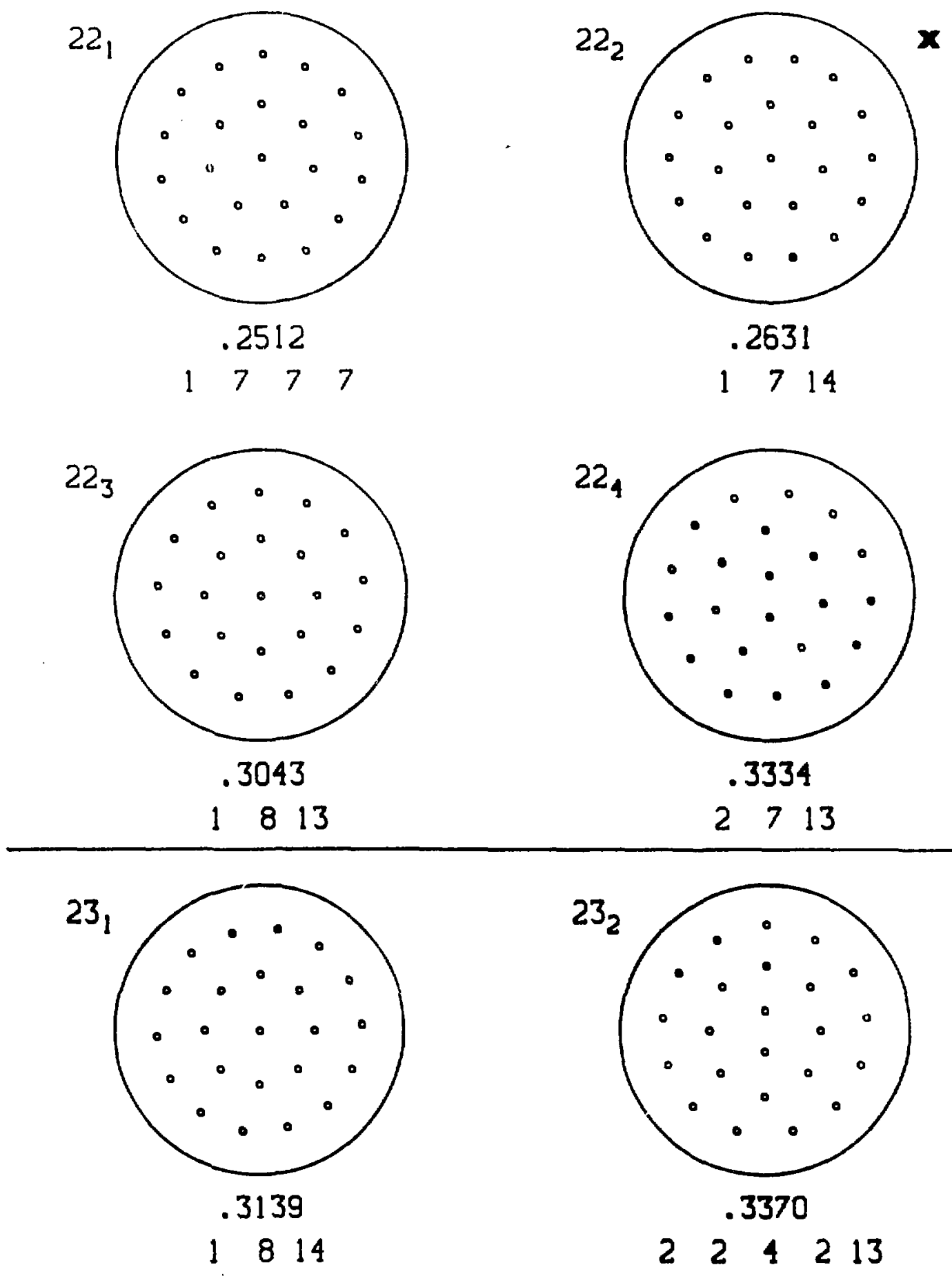

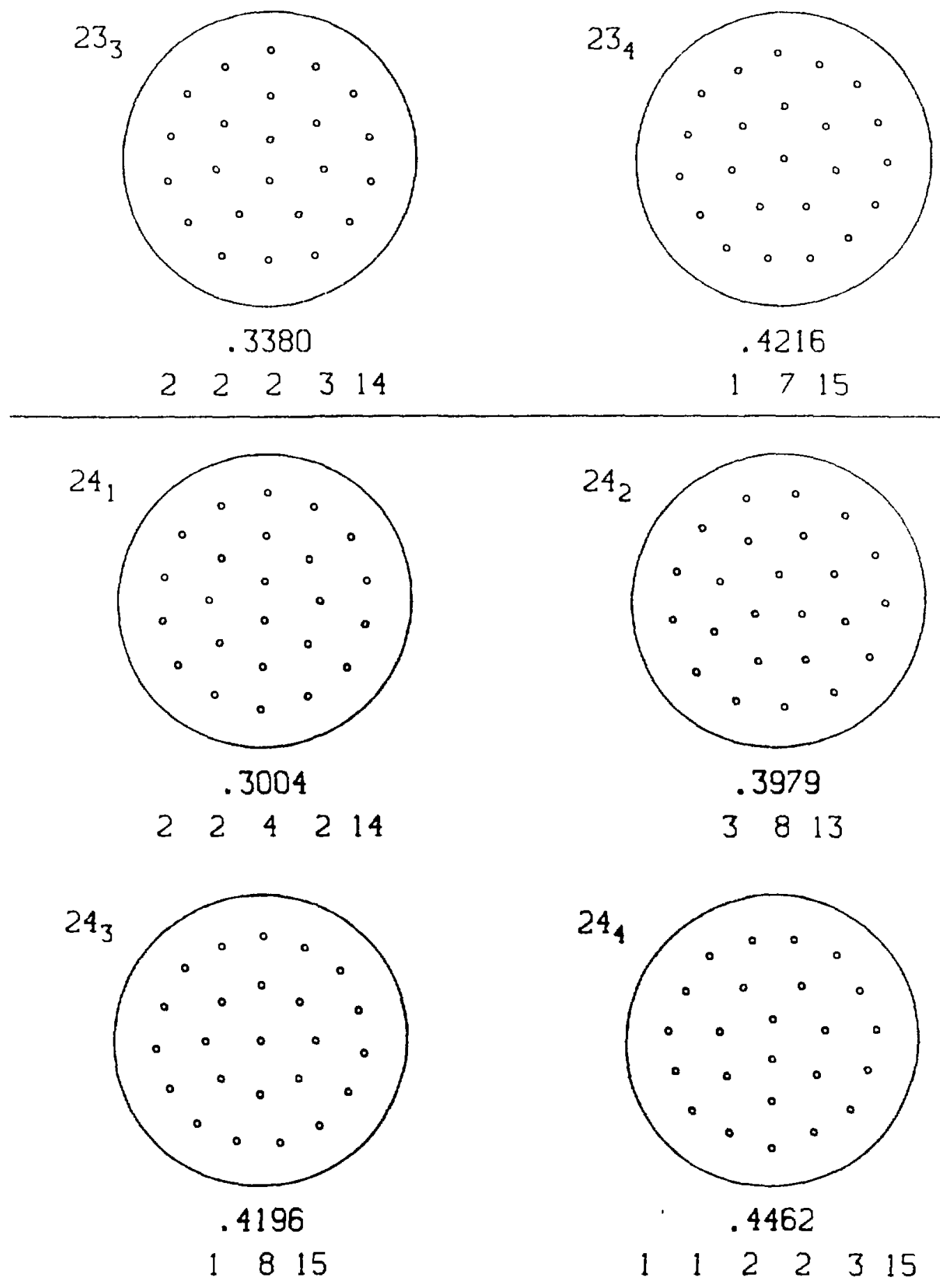

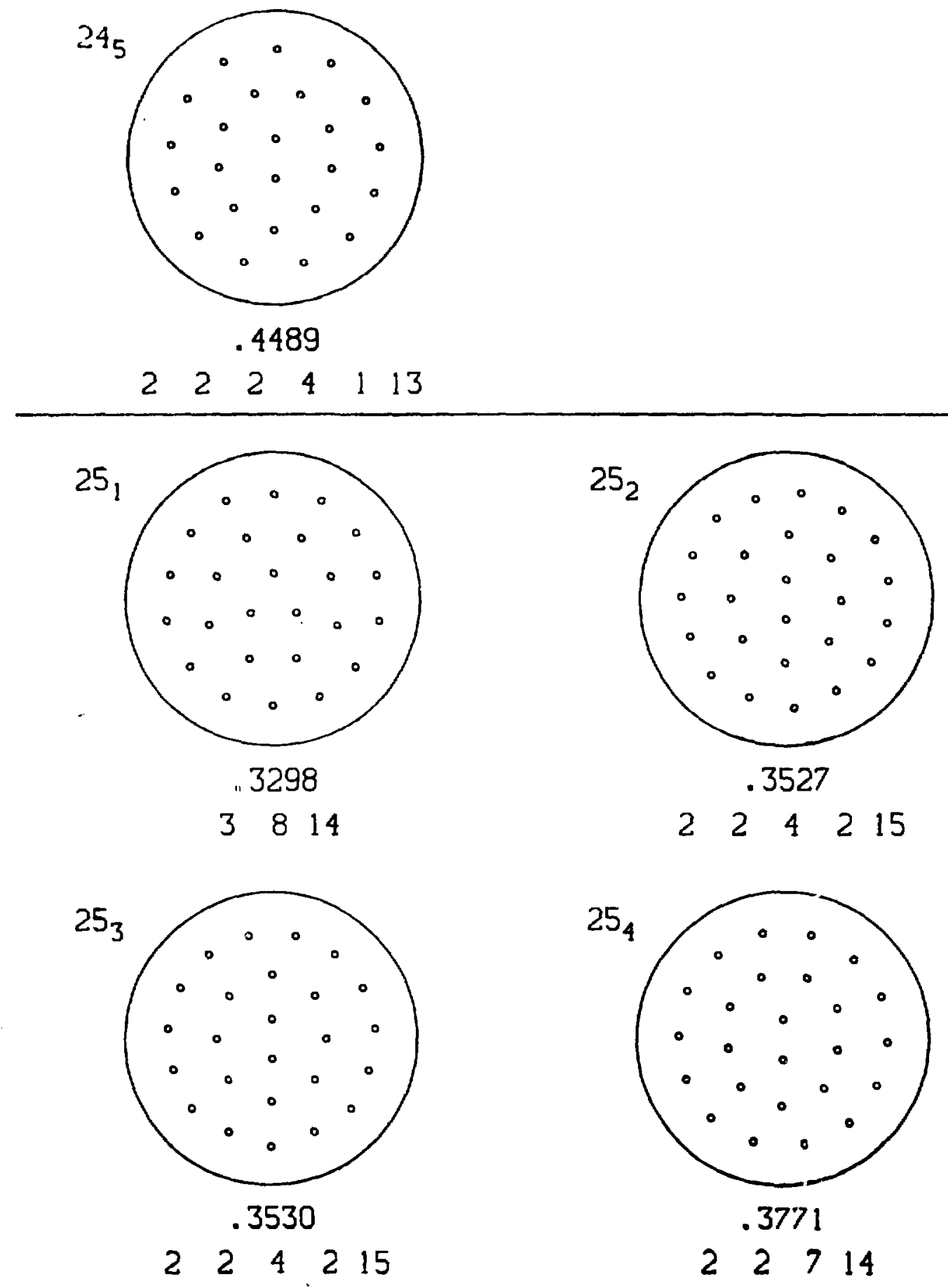

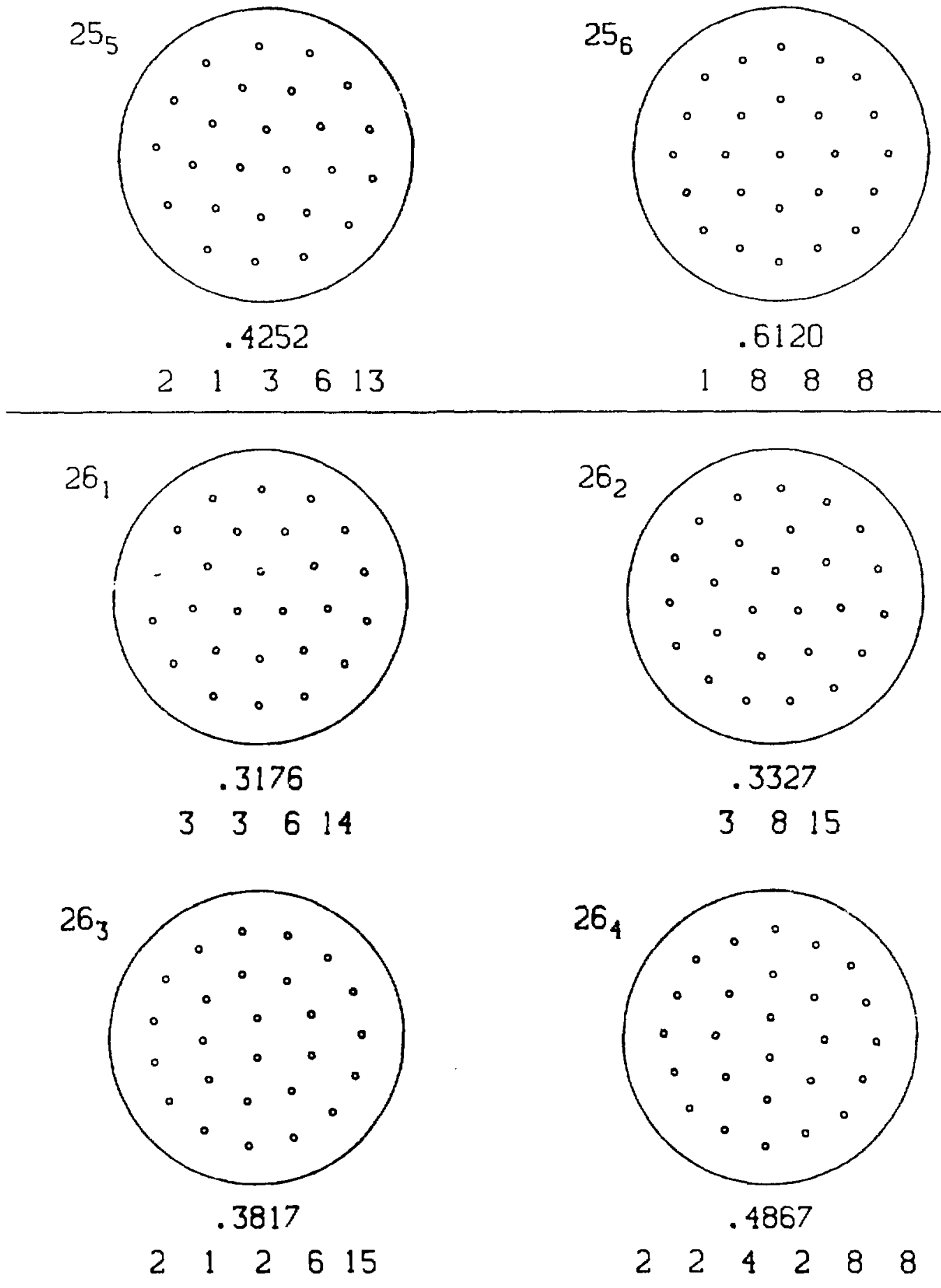

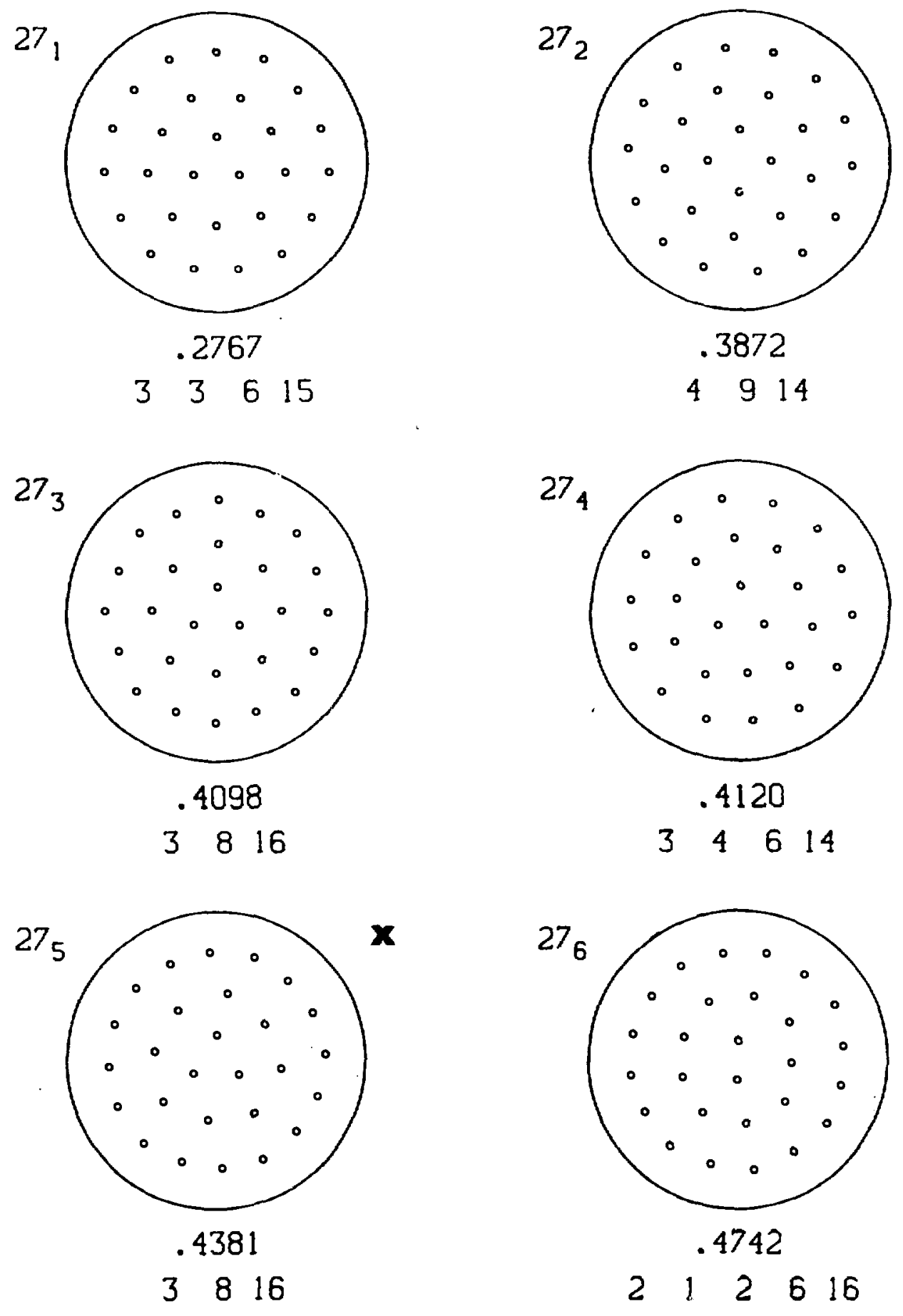

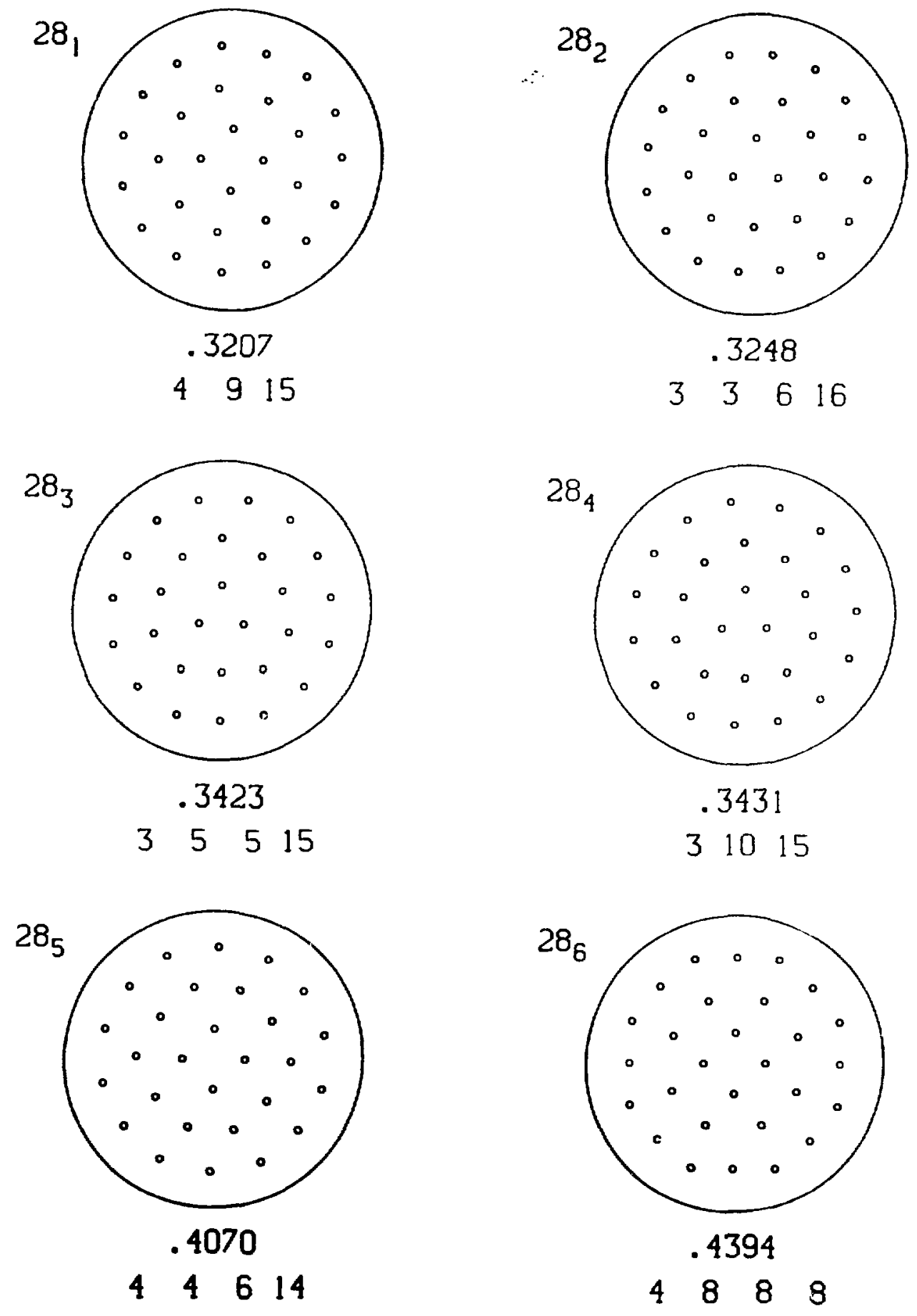

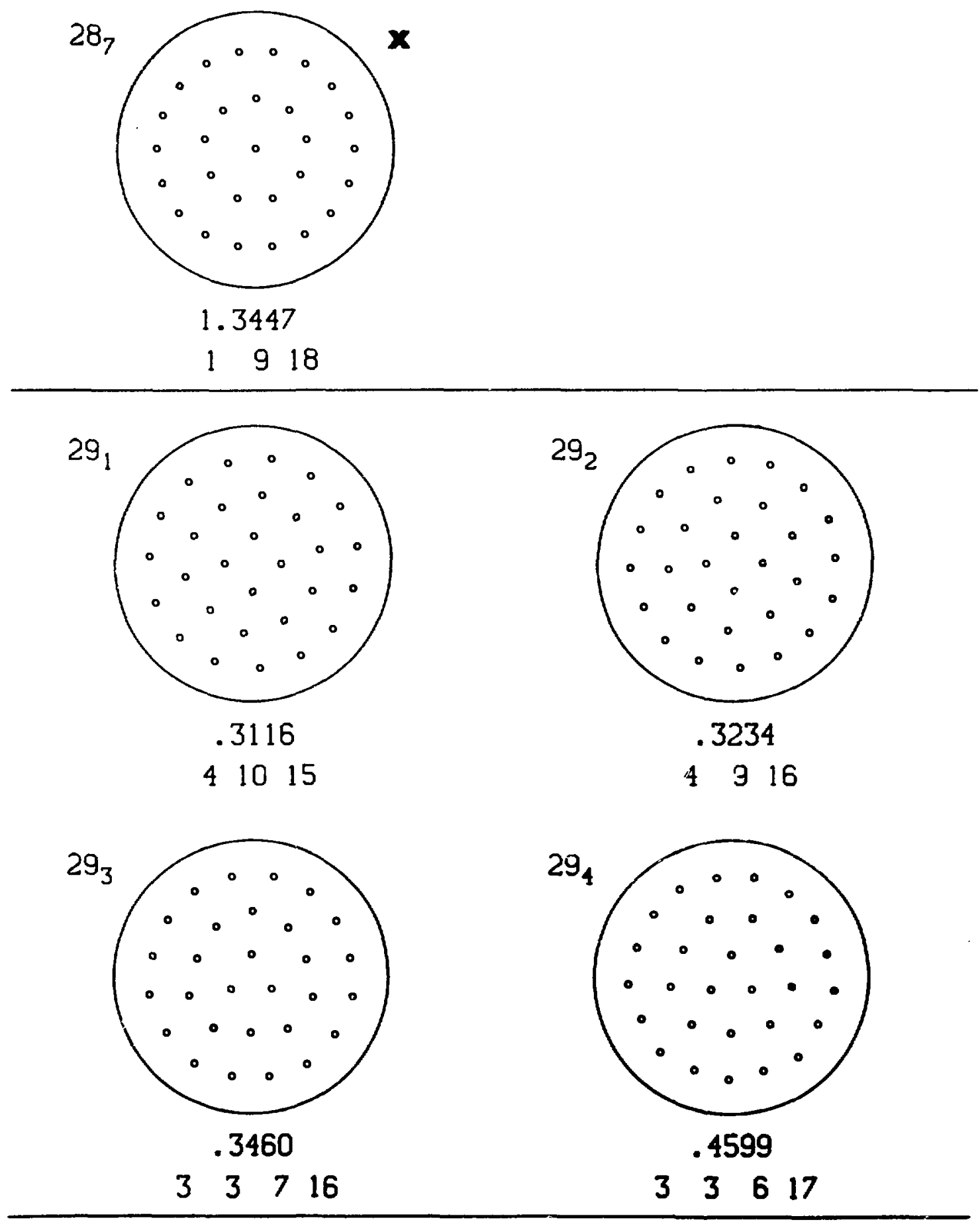
30

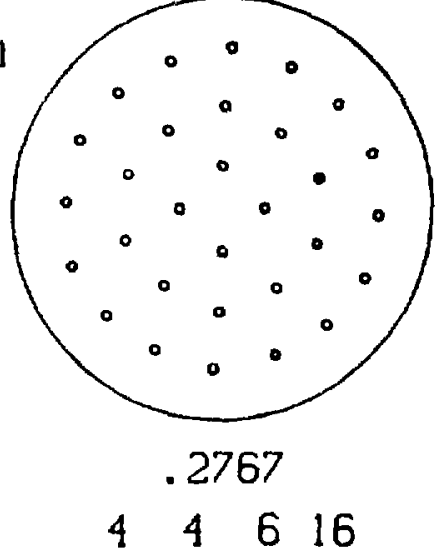

$30_{3}$

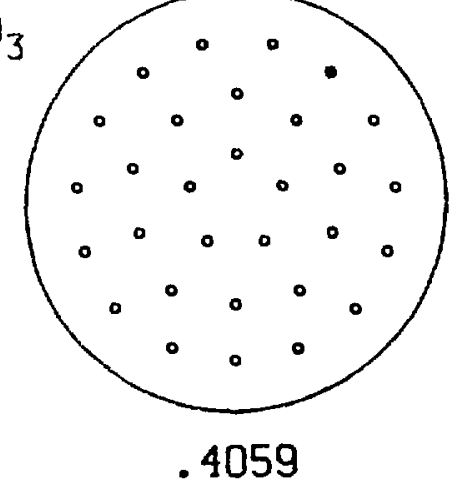

$\begin{array}{llll}5 & 5 & 5 & 15\end{array}$
$30_{2}$

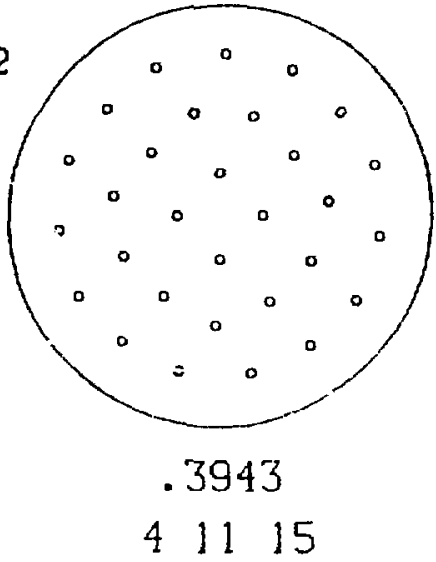

$30_{4}$

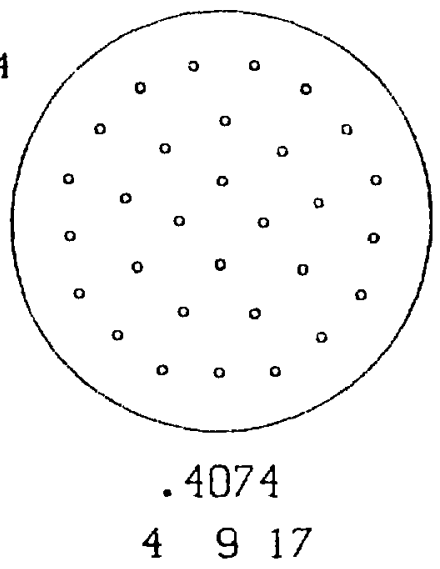

$30_{5}$

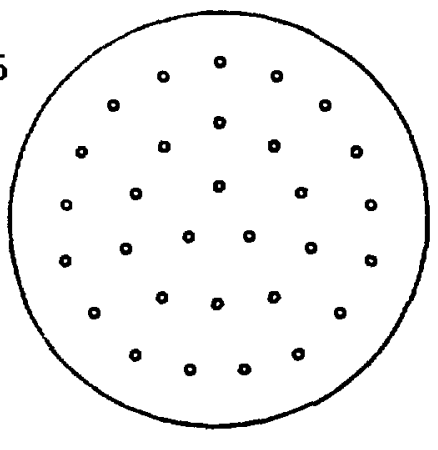

.4354

$\begin{array}{llll}3 & 3 & 7 & 17\end{array}$ 
37

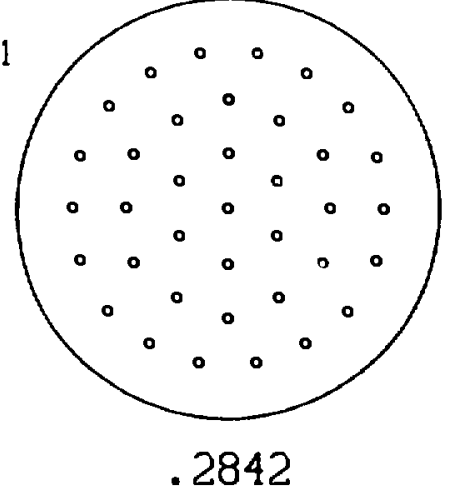

$\begin{array}{lllll}1 & 6 & 6 & 6 & 18\end{array}$

$37_{3}$

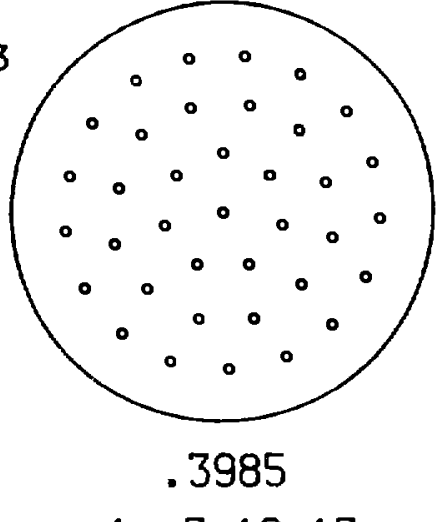

372

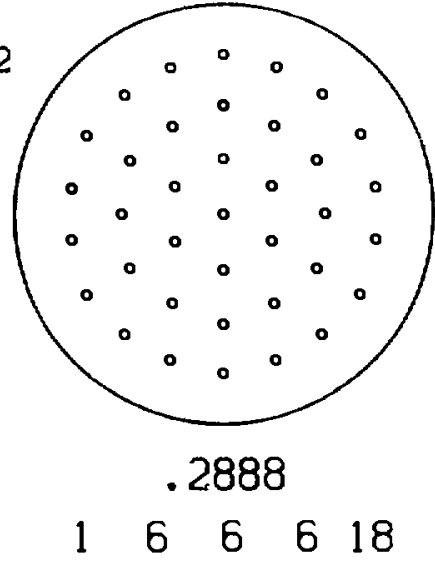

37

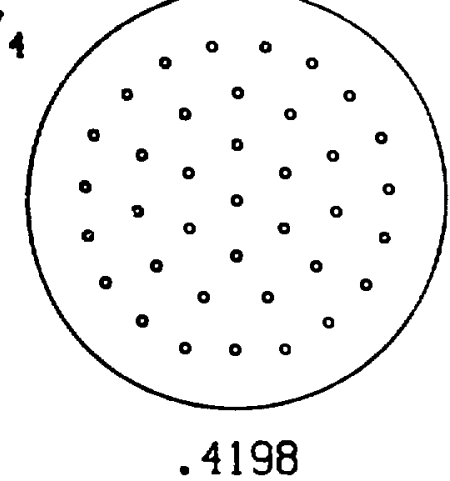

$1 \quad 6 \quad 11 \quad 19$
$50_{1}$

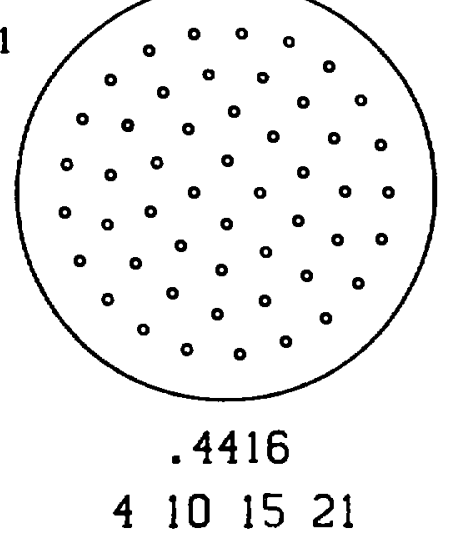

$5 \mathrm{O}_{2}$

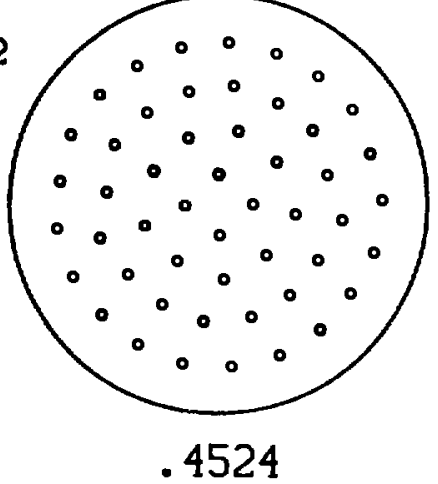

$\begin{array}{llll}4 & 9 & 16 & 21\end{array}$ 


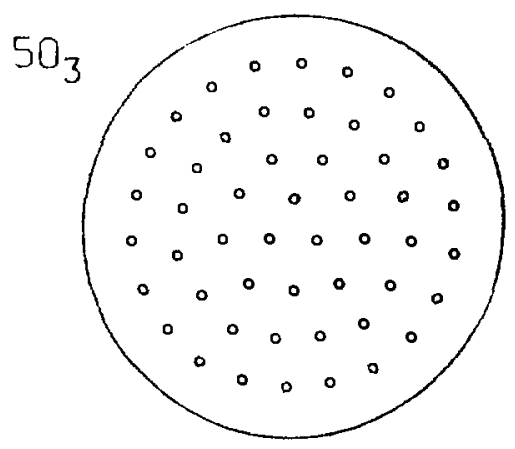

.4648

$$
\begin{array}{lllll}
3 & 3 & 6 & 16 & 22
\end{array}
$$

$50_{5}$

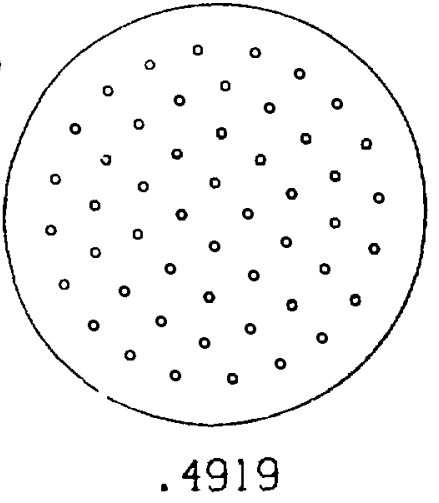

$\begin{array}{lllll}4 \quad 4 \quad 16 & 20\end{array}$

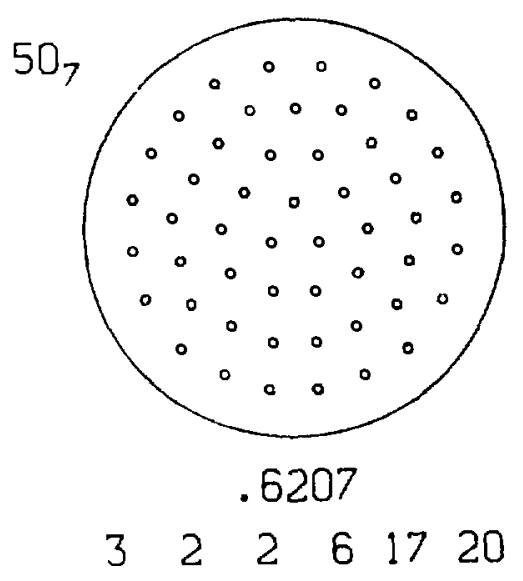

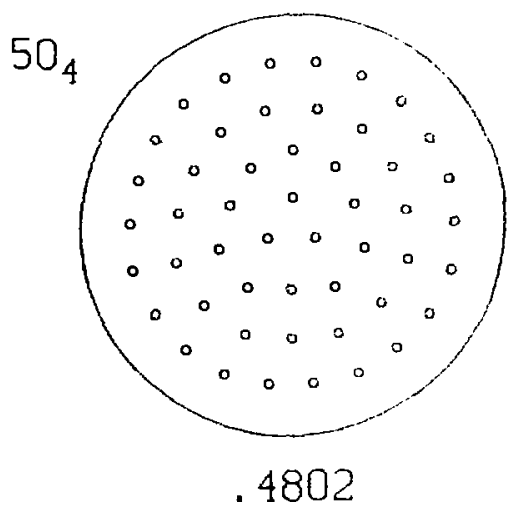

$\begin{array}{lllll}3 & 3 & 7 & 15 & 22\end{array}$

$50_{6}$

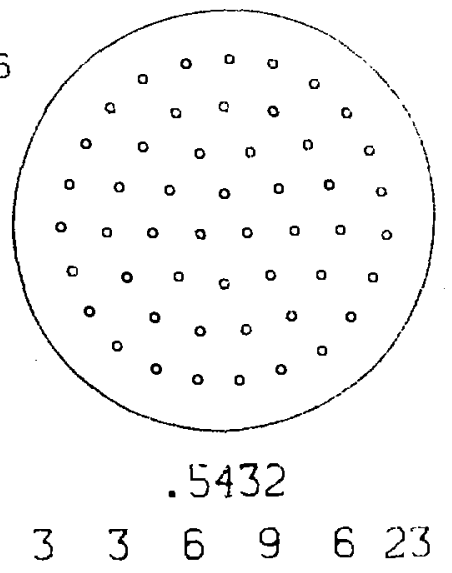


611

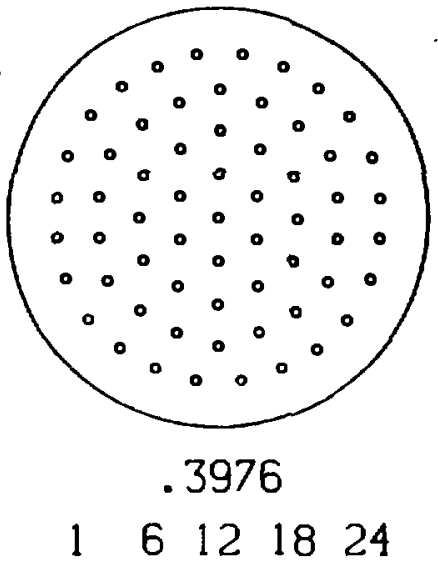

91

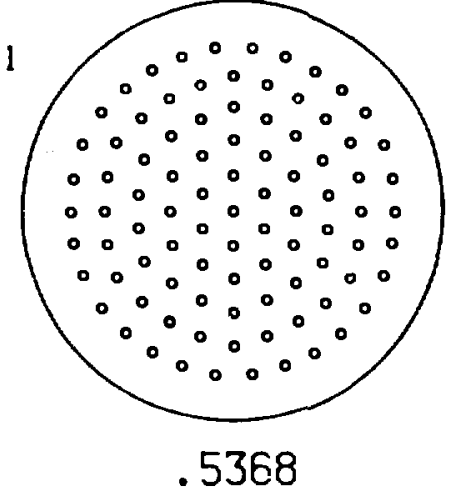

$\begin{array}{llllll}1 & 6 & 12 & 18 & 24 & 30\end{array}$
$61_{2}$

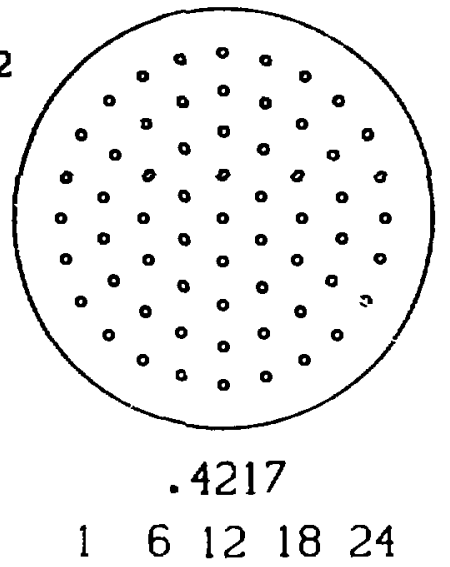

912

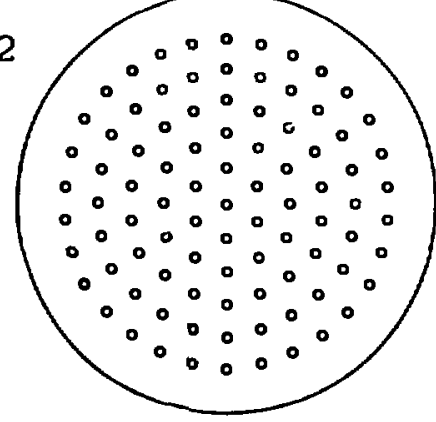

.5863

$\begin{array}{llllll}1 & 6 & 12 & 18 & 24 & 30\end{array}$
127

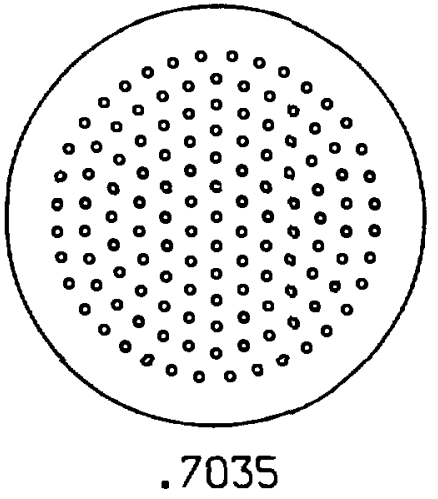

$\begin{array}{lllllll}1 & 6 & 12 & 18 & 24 & 30 & 36\end{array}$
1272

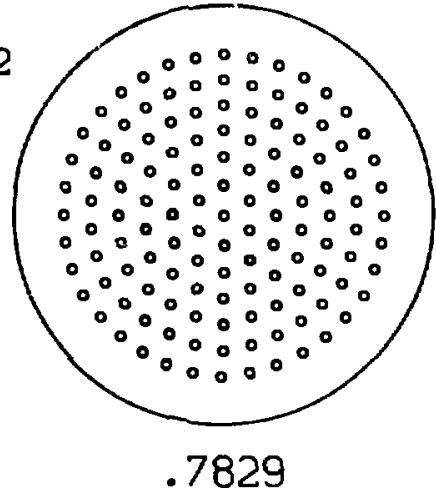

$\begin{array}{lllllll}1 & 6 & 12 & 18 & 24 & 30 & 36\end{array}$ 
169

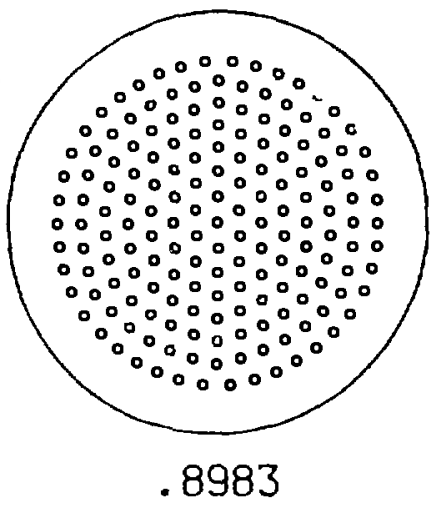

$\begin{array}{llllllll}1 & 6 & 12 & 18 & 24 & 30 & 36 & 42\end{array}$

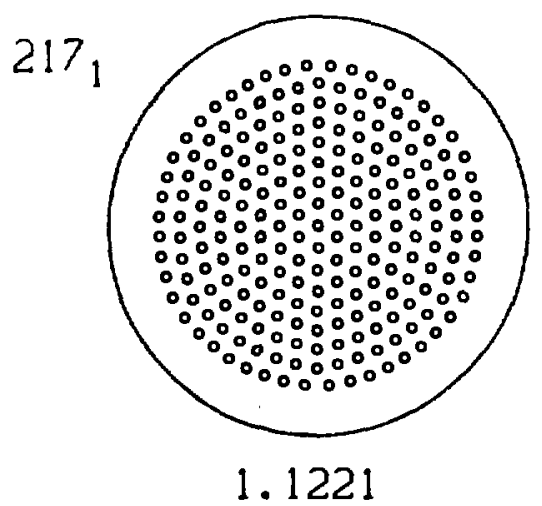

$\begin{array}{lllllllll}1 & 6 & 12 & 18 & 24 & 30 & 36 & 42 & 48\end{array}$
$169_{2}$

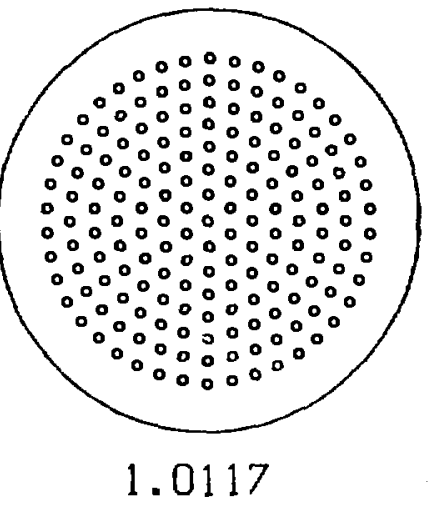

$\begin{array}{llllllll}1 & 6 & 12 & 18 & 24 & 30 & 36 & 42\end{array}$

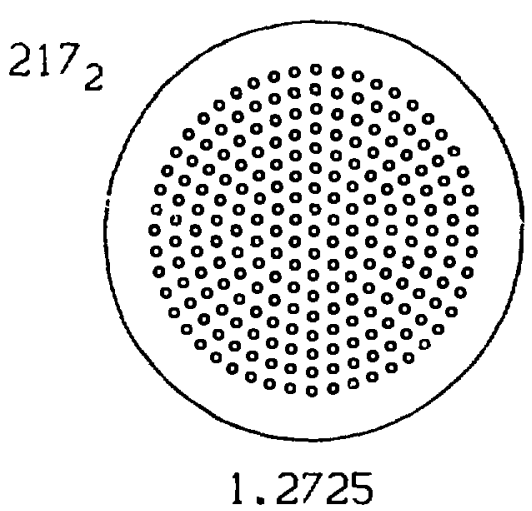

$\begin{array}{lllllllll}1 & 6 & 12 & 18 & 24 & 30 & 36 & 42 & 48\end{array}$ 


\section{REFERENCES}

1. T. H. Havelock, "Stability of Motion of Rectilinear Vortices in Ring Formation," Phil. Mag. XI, 617-633 (1931).

2. G. B. Hess, "Angular Momentum of Superfluid Helium in a Rotating Cylinder," Phys. Rev. 161, 189-193 (1967).

3. D. Stauffer and A. L. Fetter, "Distribution of Vortices in Rotating Helium II," Phys. Rev. 168, 156-159 (1968).

4. L. M. Milne-Thomson, Theoretical Hydrodynamics (Macmillan, London, 1968), Chap. XIII, §13.50.

5. H. Lamb, Hydrodynamics (Dover, Nen York, 1945), §157. 